\title{
CRANK-NICOLSON FINITE ELEMENT DISCRETIZATIONS FOR A TWO-DIMENSIONAL LINEAR SCHRÖDINGER-TYPE EQUATION POSED IN A NONCYLINDRICAL DOMAIN
}

\author{
D. C. Antonopoulou, G. D. KARAli, M. PlEXOUSAKIS, AND G. E. ZOURARIS
}

Dedicated to Professor Vassilios Dougalis on the occasion of his 65th birthday

\begin{abstract}
Motivated by the paraxial narrow-angle approximation of the Helmholtz equation in domains of variable topography, we consider an initialand boundary-value problem for a general Schrödinger-type equation posed on a two space-dimensional noncylindrical domain with mixed boundary conditions. The problem is transformed into an equivalent one posed on a rectangular domain, and we approximate its solution by a Crank-Nicolson finite element method. For the proposed numerical method, we derive an optimal order error estimate in the $L^{2}$ norm, and to support the error analysis we prove a global elliptic regularity theorem for complex elliptic boundary value problems with mixed boundary conditions. Results from numerical experiments are presented which verify the optimal order of convergence of the method.
\end{abstract}

\section{INTRODUCTION}

1.1. The physical problem. The standard narrow-angle 3D Parabolic Equation $(\mathrm{PE})$ is the Schrödinger-type equation

$$
\partial_{r} \psi=\frac{\mathrm{i}}{2 k_{0}}\left(\partial_{z}^{2} \psi+\frac{1}{r^{2}} \partial_{\theta}^{2} \psi\right)+\mathrm{i} \frac{k_{0}}{2}\left(n_{R F}^{2}-1\right) \psi
$$

that is used in the context of underwater acoustics as a paraxial and far-field approximation of the Helmholtz equation (see [19], 11]). The unknown function $\psi=\psi(r, z, \theta)$, depending on range, depth and azimuth measures the acoustic pressure in inhomogeneous, weakly range-dependent marine environments. Here, $R \geq r \geq r_{0}>0$ is the horizontal distance from a harmonic point source placed on the $z$ axis and emitting at a frequency $f_{0}$. The depth variable $z \geq 0$ is increasing downwards while the azimuth $\theta$ varies in the interval $\left[\theta_{A}, \theta_{B}\right]$ with $0 \leq \theta_{A}<\theta_{B} \leq 2 \pi$. Also, $k_{0}:=\frac{2 \pi f_{0}}{c_{0}}$ is a reference wave number where the constant $c_{0}$ is a reference sound speed, and $n_{R F}(r, z, \theta):=\frac{c_{0}}{c(r, z, \theta)}$ is the refraction index where $c(r, z, \theta)$ is the sound speed in the water column. The variable bottom topography is identified in cylindrical coordinates by a positive surface $z=s(r, \theta)$. Thus, the equation (1.1) is posed in a noncylindrical domain $\mathcal{S}:=\bigcup_{r \in\left[r_{0}, R\right]} \Omega(r)$

Received by the editor September 1, 2011 and, in revised form, October 29, 2012 and October 3, 2013.

2000 Mathematics Subject Classification. Primary 65M12, 65M15, 65M60.

Key words and phrases. Schrödinger-type equation, noncylindrical domain, Robin-type boundary condition, elliptic regularity, Crank-Nicolson time stepping, finite element method, apriori error estimates, underwater acoustics. 
where

$$
\Omega(r):=\left\{(z, \theta) \in \mathbb{R}^{2}: \theta \in\left[\theta_{A}, \theta_{B}\right], z \in[0, s(r, \theta)]\right\}
$$

is a range-dependent domain with boundary $\partial \Omega(r)=\bigcup_{i=1}^{4} \omega_{i}(r)$ with (cf. Figure 1)

$$
\begin{aligned}
& \omega_{1}(r):=\left\{(0, \theta) \in \mathbb{R}^{2}: \theta \in\left[\theta_{A}, \theta_{B}\right]\right\}, \\
& \omega_{2}(r):=\left\{\left(z, \theta_{A}\right) \in \mathbb{R}^{2}: z \in\left[0, s\left(r, \theta_{A}\right)\right]\right\}, \\
& \omega_{3}(r):=\left\{(s(r, \theta), \theta) \in \mathbb{R}^{2}: \theta \in\left[\theta_{A}, \theta_{B}\right]\right\}, \\
& \omega_{4}(r):=\left\{\left(z, \theta_{B}\right) \in \mathbb{R}^{2}: z \in\left[0, s\left(r, \theta_{B}\right)\right]\right\} .
\end{aligned}
$$

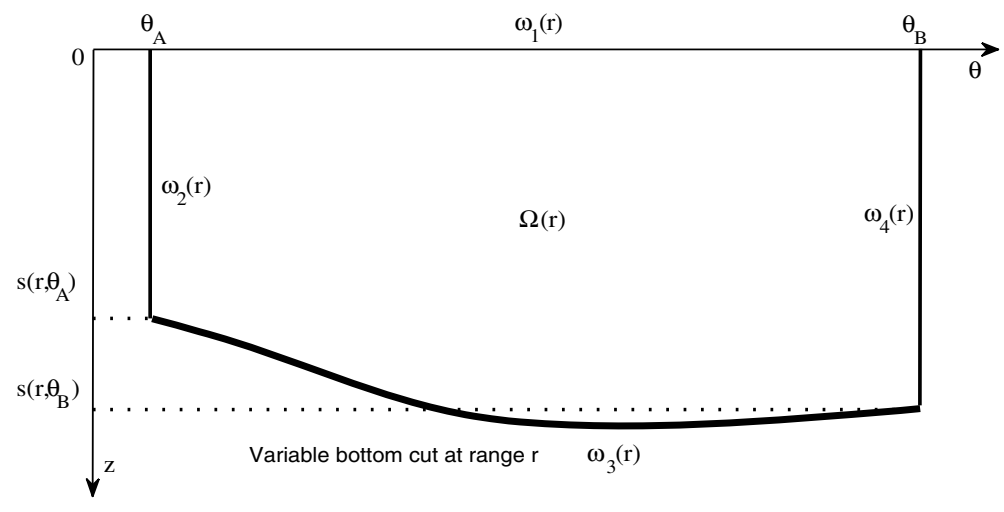

Figure 1. The range dependent domain $\Omega(r)$.

To formulate an initial and boundary value problem (ibvp) for equation (1.1), first we impose an initial condition

$$
\psi\left(r_{0}, z, \theta\right)=\psi_{0}(z, \theta) \quad \forall(z, \theta) \in \Omega\left(r_{0}\right),
$$

where $\psi_{0}$ is a representation of the harmonic point source and usually is a function with compact support (see [19]). Then, we impose boundary conditions on $\partial \Omega(r)$ which depend on the physical assumptions we make for the sea environment. In particular, the horizontal sea surface and the lateral boundaries are assumed to be perfectly absorbing, which is mathematically modeled by imposing the following pressure release condition:

$$
\psi(r, z, \theta)=0 \quad \forall(z, \theta) \in \omega_{1}(r) \cup \omega_{2}(r) \cup \omega_{4}(r) .
$$

The bottom is assumed to be acoustically rigid, that is, mathematically modeled by imposing the Neumann boundary condition $\frac{\partial \psi}{\partial n}(r, \cdot)=0$ on $\omega_{3}(r)$, i.e.,

(1.4) $\partial_{z} \psi(r, z, \theta)-\frac{1}{r^{2}} \partial_{\theta} s(r, \theta) \partial_{\theta} \psi(r, z, \theta)=\partial_{r} s(r, \theta) \partial_{r} \psi(r, z, \theta) \quad \forall(z, \theta) \in \omega_{3}(r)$.

The boundary condition (1.4) is a natural boundary condition for the Helmholtz equation, but for the Schrödinger-type equation (1.1) is a nonstandard dynamical boundary condition since it includes the term $\partial_{r} \psi$ and $r$ is a time-like variable. Abrahamsson and Kreiss consider the ibvp (1.1)-(1.4) in the axial symmetric case (where there is no dependence on $\theta$ ) and prove that it is well-posed when the bottom is strictly monotone (see [1]). Thus, the well-posedness of the problem in the general case of a smooth bottom that changes slope is not guaranteed something indicated by systematic numerical simulations reported in [2, 7] which show that 
significant instabilities may develop. Also, recent results in 21] show that the heat equation in two space dimensions has no weak solution when the coefficient of the evolution derivative in the dynamical boundary condition is positive. The authors of [1] and [2] also observed that in certain downsloping environments the amplitude of higher order modes increases when range increases instead of having a low contribution in the propagating sound. This is evidence that (1.4) is not always a physically correct boundary condition for equation (1.1) which gave them the motivation to derive an alternative boundary condition that in axial symmetric environments simulates in a satisfactory way the reflection of a plane wave over a rigid downsloping bottom with constant slope (see [2]). Sturm in [18] extends the boundary condition of Abrahamsson-Kreiss for general 3D environments using the paraxial assumption included in the derivation of (1.1) as an approximate equation to the Helmholtz equation. This alternative boundary condition will be our bottom boundary condition and is formulated as follows:

$$
\partial_{z} \psi(r, z, \theta)-\frac{1}{r^{2}} \partial_{\theta} s(r, \theta) \partial_{\theta} \psi(r, z, \theta)=\mathrm{i} k_{0} \partial_{r} s(r, \theta) \psi(r, z, \theta) \forall(z, \theta) \in \omega_{3}(r) .
$$

Remark 1.1. For $r \in\left[r_{0}, R\right]$, let $D(r)$ be a real, $2 \times 2$ diagonal matrix with $D_{11}(r):=$ $\frac{1}{2 k_{0}}$ and $D_{22}(r):=\frac{1}{2 k_{0} r^{2}}$. Observing that the outward-pointing unit normal vector on $\omega_{3}(r)$ is given by $\mathbf{n}_{\omega_{3}(r)}(z, \theta)=\frac{\left(1,-\partial_{\theta} s(r, \theta)\right)}{\left[1+\left(\partial_{\theta} s(r, \theta)\right)^{2}\right]^{\frac{1}{2}}}$, it is easily verified that the boundary condition (1.5) is written equivalently as

$$
\mathbf{n}_{\omega_{3}(r)}^{T}(z, \theta) D(r) \nabla \psi(r, z, \theta)=\frac{i}{2} \frac{\partial_{r} s(r, \theta)}{\left[1+\left(\partial_{\theta} s(r, \theta)\right)^{2}\right]^{\frac{1}{2}}} \psi(r, z, \theta) \quad \forall(z, \theta) \in \omega_{3}(r),
$$

which indicates that (1.5) is a Robin-type boundary condition for (1.1).

Remark 1.2. Let $\psi$ be the solution of the ibvp (1.1)-(1.3) and (1.5). Multiplying both sides of (1.1) with $\bar{\psi}$, and then integrating both sides on $\Omega(r)$, taking real parts and using integration by parts we conclude that $\int_{\Omega(r)}|\psi(r, z, \theta)|^{2} d z d \theta=$ $\int_{\Omega\left(r_{0}\right)}\left|\psi_{0}(z, \theta)\right|^{2} d z d \theta$ for $r \in\left[r_{0}, R\right]$.

1.2. A transformation of the physical problem. For $\psi$ being the solution of the ibvp (1.1)-(1.3) and (1.5), we introduce (cf. [5, 6, 18, 22, ) the function

$$
v(r, y, \theta):=\sqrt{s(r, \theta)} \psi(r, y s(r, \theta), \theta) \quad \forall(r, y, \theta) \in\left[r_{0}, R\right] \times[0,1] \times\left[\theta_{A}, \theta_{B}\right]
$$

and simplify the notation in (1.1) by setting $\gamma_{R F}(r, z, \theta):=\frac{k_{0}}{2}\left(n_{R F}^{2}(r, z, \theta)-1\right)$ and $\alpha:=\frac{1}{2 k_{0}}$. Then, it is easily seen that $v$ is specified as the solution of the following ibvp:

$$
\begin{array}{ll}
\partial_{r} v=\mathrm{i} \operatorname{div}(\mathfrak{D}(r, y, \theta) \nabla v)+y \frac{\partial_{r} s(r, \theta)}{s(r, \theta)} \partial_{y} v+\mathrm{i} \gamma(r, y, \theta) v \\
& \forall(r, y, \theta) \in\left[r_{0}, R\right] \times \overline{\mathcal{D}}, \\
v(r, y, \theta)=0 & \forall(r, y, \theta) \in\left[r_{0}, R\right] \times \Gamma_{\mathrm{D}}, \\
\mathbf{n}^{T} \mathfrak{D}(r, y, \theta) \nabla v(r, y, \theta)=\mathrm{i} \gamma_{\mathrm{BC}}(r, \theta) v(r, y, \theta) & \forall(r, y, \theta) \in\left[r_{0}, R\right] \times \Gamma_{\mathrm{R}}, \\
v\left(r_{0}, y, \theta\right)=v_{0}(y, \theta) & \forall(y, \theta) \in \overline{\mathcal{D}},
\end{array}
$$

where: $\mathcal{D}:=(0,1) \times\left(\theta_{A}, \theta_{B}\right), \mathbf{n}:=(1,0)^{T}, \Gamma_{\mathrm{R}}:=\left\{(1, \theta) \in \mathbb{R}^{2}: \theta \in\left[\theta_{A}, \theta_{B}\right]\right\}$ and $\Gamma_{\mathrm{D}}:=\left\{(0, \theta) \in \mathbb{R}^{2}: \quad \theta \in\left[\theta_{A}, \theta_{B}\right]\right\} \cup\left\{(y, \theta) \in \mathbb{R}^{2}: \quad y \in\{0,1\}\right.$ and $\left.\theta \in\left[\theta_{A}, \theta_{B}\right]\right\}$, 
$\mathfrak{D}:\left[r_{0}, R\right] \times \overline{\mathcal{D}} \rightarrow \mathbb{R}^{2 \times 2}$ with $\mathfrak{D}_{11}(r, y, \theta):=\frac{\alpha}{s^{2}(r, \theta)}+\frac{\alpha}{r^{2}} y^{2}\left[\frac{\partial_{\theta} s(r, \theta)}{s(r, \theta)}\right]^{2}, \mathfrak{D}_{22}(r, y, \theta):=$ $\frac{\alpha}{r^{2}}$ and $\mathfrak{D}_{12}(r, y, \theta)=\mathfrak{D}_{21}(r, y, \theta):=-\frac{\alpha}{r^{2}} y \frac{\partial_{\theta} s(r, \theta)}{s(r, \theta)}, v_{0}: \overline{\mathcal{D}} \rightarrow \mathbb{C}$ with $v_{0}(y, \theta):=$ $\sqrt{s\left(r_{0}, \theta\right)} \psi_{0}\left(y s\left(r_{0}, \theta\right), \theta\right), \gamma_{\mathrm{BC}}:\left[r_{0}, R\right] \times\left[\theta_{A}, \theta_{B}\right]$ with

$$
\gamma_{\mathrm{BC}}(r, \theta):=\frac{\partial_{r} s(r, \theta)}{2 s(r, \theta)}+\mathrm{i} \frac{\alpha}{2 r^{2}}\left(\frac{s_{\theta}(r, \theta)}{s(r, \theta)}\right)^{2}
$$

and $\gamma:\left[r_{0}, R\right] \times \overline{\mathcal{D}} \rightarrow \mathcal{C}$ with

$$
\gamma(r, y, \theta):=\gamma_{R F}(r, y s(r, \theta), \theta)+\frac{\alpha}{r^{2}} \frac{3\left(\partial_{\theta} s(r, \theta)\right)^{2}-2 s(r, \theta) \partial_{\theta}^{2} s(r, \theta)}{4 s^{2}(r, \theta)}-\mathrm{i} \frac{\partial_{r} s(r, \theta)}{2 s(r, \theta)} .
$$

We note that, for $(r, y, \theta) \in\left[r_{0}, R\right] \times \overline{\mathcal{D}}$, the matrix $\mathfrak{D}(r, y, \theta)$ is positive definite since it holds that

$$
x^{T} \mathfrak{D}(r, y, \theta) x \geq \frac{\alpha}{2 r^{2}}\left(x_{1}\right)^{2}+\frac{\alpha}{2\left[r^{2}+\alpha y^{2}\left(\partial_{\theta} s(r, \theta)\right)^{2}\right]}\left(x_{2}\right)^{2} \quad \forall x \in \mathbb{R}^{2} .
$$

Remark 1.3. The transformation (1.6) we use here combines a change of variable with the multiplication with $\sqrt{s}$ that makes the solution $v$ of the problem $L^{2}$ conservative (cf. Remark 1.2), i.e. $\int_{\mathcal{D}}|v(r, y, \theta)|^{2} d y d \theta=\int_{\mathcal{D}}\left|v_{0}(y, \theta)\right|^{2} d y d \theta$ for $r \in\left[r_{0}, R\right]$.

1.3. A generalized problem. Motivated by the formulation of the physical problem (1.7) and for the sake of a more general mathematical setting, in the sequel we will work with the following ibvp:

$$
\begin{array}{ll}
\partial_{r} u=\mathrm{i} \operatorname{div}(A(r, y, \theta) \nabla u)+b(r, y, \theta) \nabla u+\mathrm{i} \beta(r, y, \theta) u+f(r, y, \theta) \\
& \forall(r, y, \theta) \in\left[r_{0}, R\right] \times \overline{\mathcal{D}}, \\
(1.8) \quad u(r, y, \theta)=0 & \forall(r, y, \theta) \in\left[r_{0}, R\right] \times \Gamma_{\mathrm{D}}, \\
\mathbf{n}^{T} A(r, y, \theta) \nabla u(r, y, \theta)=\mathrm{i} \lambda_{\mathrm{BC}}(r, \theta) u(r, y, \theta) & \forall(r, y, \theta) \in\left[r_{0}, R\right] \times \Gamma_{\mathrm{R}}, \\
u\left(r_{0}, y, \theta\right)=u_{0}(y, \theta) & \forall(y, \theta) \in \overline{\mathcal{D}} .
\end{array}
$$

Here, $u_{0}: \overline{\mathcal{D}} \rightarrow \mathbb{C}, \beta:\left[r_{0}, R\right] \times \overline{\mathcal{D}} \rightarrow \mathbb{C}, f:\left[r_{0}, R\right] \times \overline{\mathcal{D}} \rightarrow \mathbb{C}$ and $\lambda_{\mathrm{BC}}:\left[r_{0}, R\right] \times$ $\left[\theta_{A}, \theta_{B}\right] \rightarrow \mathbb{C}$ are smooth complex valued functions. Also, $b:\left[r_{0}, R\right] \times \overline{\mathcal{D}} \rightarrow \mathbb{R}^{2}$ is a real vector valued function with smooth components satisfying

$$
b_{1}(r, 1, \theta)-2 \operatorname{Re}\left(\lambda_{\mathrm{BC}}(r, \theta)\right) \leq 0 \quad \forall(r, \theta) \in\left[r_{0}, R\right] \times\left[\theta_{A}, \theta_{B}\right],
$$

and $A:\left[r_{0}, R\right] \times \overline{\mathcal{D}} \rightarrow \mathbb{R}^{2 \times 2}$ is a real matrix valued function with smooth components for which we assume that $A(r, y, \theta)$ is symmetric for all $(r, y, \theta) \in\left[r_{0}, R\right] \times \overline{\mathcal{D}}$ and there exists a positive constant $C_{\star}$ such that

$$
x^{T} A(r, y, \theta) x \geq C_{\star}|x|^{2} \quad \forall x \in \mathbb{R}^{2}, \quad \forall(r, y, \theta) \in\left[r_{0}, R\right] \times \overline{\mathcal{D}} .
$$

Finally, we assume that there exists a function $\lambda_{\mathrm{G}} \in C^{1}\left(\left[r_{0}, R\right] \times \overline{\mathcal{D}} ; \mathbb{C}\right)$ such that

$$
\left.\lambda_{\mathrm{G}}(r, y, \theta)\right|_{\Gamma_{\mathrm{R}}}=\lambda_{\mathrm{BC}}(r, \theta) \quad \forall(r, y, \theta) \in\left[r_{0}, R\right] \times \Gamma_{\mathrm{R}} .
$$

For the rest of the paper we make the general assumption that the data are smooth enough and compatible so that (1.8) admits a solution regular enough for our purposes.

Remark 1.4. The physical ibvp (1.7) is a special case of the problem (1.8), where the equality holds in (1.9). 
Remark 1.5. The solution $u$ of (1.8) is $L^{2}$-conservative when $f=0$, the equality holds in (1.9) and $2 \operatorname{Im}(\beta)+\operatorname{div}(b)=0$. All these conditions are satisfied by the data of the physical problem (1.7).

1.4. Function spaces and norms. We denote by $L^{2}(\mathcal{D})$ the space of the Lebesgue measurable, complex valued functions which are square integrable on $\mathcal{D}$ with respect to Lebesgue's measure, provided with the standard norm $\|g\|:=\left(\int_{\mathcal{D}}|g(y, \theta)|^{2} d y d \theta\right)^{\frac{1}{2}}$ for $g \in L^{2}(\mathcal{D})$. The standard inner product in $L^{2}(\mathcal{D})$ that produces the norm $\|\cdot\|$ is written as $(\cdot, \cdot)$, i.e., $\left(g_{1}, g_{2}\right):=\int_{\mathcal{D}} g_{1}(y, \theta) \overline{g_{2}(y, \theta)} d y d \theta$ for $g_{1}, g_{2} \in L^{2}(\mathcal{D})$. Let $\mathbb{N}$ be the set of all positive integers and $\mathbb{N}_{0}:=\mathbb{N} \cup\{0\}$. For $s \in \mathbb{N}_{0}, H^{s}(\mathcal{D})$ will be the Sobolev space of complex valued functions having generalized derivatives up to order $s$ in the space $L^{2}(\mathcal{D})$, and by $\|\cdot\|_{s}$ we denote its usual norm, i.e. $\|g\|_{s}:=\left(\sum_{\alpha \in \mathbb{N}_{0}^{2},|\alpha| \leq s}\left\|\partial^{\alpha} g\right\|_{\mathcal{D}}^{2}\right)^{\frac{1}{2}}$ for $g \in H^{s}(\mathcal{D})$.

Let $\omega$ be a piecewise Lipschitz subset of $\partial \mathcal{D}$. Then, we denote by $L^{2}(\omega)$ the space of complex valued functions which are measurable and square integrable on $\omega$ with respect to the one-dimensional surface measure induced by the Lebesgue measure, and is provided by the standard norm $|g|_{\omega}:=\left(\int_{\omega}|g|^{2} d S\right)^{\frac{1}{2}}$ for $g \in L^{2}(\omega)$. The standard inner product in $L^{2}(\omega)$ that produces the norm $|\cdot|_{\omega}$ is defined by $\left\langle v_{a}, v_{b}\right\rangle_{\omega}:=\int_{\omega} v_{a} \bar{v}_{b} d S$ for $v_{a}, v_{b} \in L^{2}(\omega)$. Also, we denote by $\tau_{\partial \mathcal{D}}: H^{1}(\mathcal{D}) \rightarrow$ $L^{2}(\partial \mathcal{D})$ the well-known trace operator which is bounded, i.e. there exists a positive constant $C_{1, \mathrm{TR}}$ such that

$$
\left|\tau_{\partial \mathcal{D}} w\right|_{\partial \mathcal{D}} \leq C_{1, \mathrm{TR}}\|w\|_{1} \quad \forall w \in H^{1}(\mathcal{D}) .
$$

By $\mathbb{H}^{1}(\mathcal{D})$ we denote the subspace of $H^{1}(\mathcal{D})$ consisting of functions which vanish at $\Gamma_{\mathrm{D}}$ in the sense of trace, i.e., $\mathbb{H}^{1}(\mathcal{D}):=\left\{w \in H^{1}(\mathcal{D}):\left.\tau_{\partial \mathcal{D}} w\right|_{\Gamma_{\mathrm{D}}}=0\right\}$. Then, we set $\mathbb{H}^{s}(\mathcal{D}):=\mathbb{H}^{1}(\mathcal{D}) \cap H^{s}(\mathcal{D})$ for $s \in \mathbb{N}$. It is well-known that there exists a positive constant $C_{2, \mathrm{TR}}$ such that

$$
\left|\tau_{\partial \mathcal{D}} w\right|_{\partial \mathcal{D}} \leq C_{2, \mathrm{TR}}\|w\|^{\frac{1}{2}}\|\nabla w\|^{\frac{1}{2}} \quad \forall w \in \mathbb{H}^{1}(\mathcal{D}) .
$$

Also, we define the space $H^{\frac{1}{2}}\left(\Gamma_{\mathrm{R}}\right):=\left\{\left.\tau_{\partial \mathcal{D}} w\right|_{\Gamma_{\mathrm{R}}}: w \in \mathbb{H}^{1}(\mathcal{D})\right\}$ provided with the norm

$$
|g|_{\frac{1}{2}, \Gamma_{\mathrm{R}}}:=\inf \left\{\|w\|_{1}: w \in \mathbb{H}^{1}(\mathcal{D}) \text { and }\left.\tau_{\partial \mathcal{D}} w\right|_{\Gamma_{\mathrm{R}}}=g\right\}
$$

and, for $g \in L^{2}\left(\Gamma_{\mathrm{R}}\right)$, we define the negative norm

$$
|g|_{-\frac{1}{2}, \Gamma_{\mathrm{R}}}:=\sup \left\{\frac{\left|\left\langle g, \tau_{\partial \mathcal{D}} w\right\rangle_{\Gamma_{\mathrm{R}}}\right|}{\|w\|_{1}}: w \in \mathbb{H}^{1}(\mathcal{D}) \text { and } w \neq 0\right\} \text {. }
$$

1.5. A weak formulation of (1.8). Taking formally the $L^{2}(\mathcal{D})$ inner product of both sides of the partial differential equation in (1.8) by $\phi \in \mathbb{H}^{1}(\mathcal{D})$, and then integrating by parts and using the boundary conditions, we arrive at

$$
\left(\partial_{r} u(r), \phi\right)=-\mathrm{i} \mathcal{G}(r ; u(r), \phi)+\mathrm{i}(\beta(r) u(r), \phi)+(f(r), \phi) \quad \forall r \in\left[r_{0}, R\right],
$$

where, for $r \in\left[r_{0}, R\right], \mathcal{G}(r ; \cdot, \cdot): H^{1}(\mathcal{D}) \times H^{1}(\mathcal{D}) \rightarrow \mathbb{C}$ is a sesquilinear form given by

$$
\mathcal{G}(r ; v, w):=(A(r) \nabla v, \nabla w)-\mathrm{i}\left\langle\lambda_{\mathrm{BC}}(r) v, w\right\rangle_{\Gamma_{\mathrm{R}}}+\mathrm{i}(b(r) \nabla v, w) \quad \forall v, w \in H^{1}(\mathcal{D}) .
$$


Proposition 1.6. The weak problem (1.14) has at most one solution,

$$
u \in C^{1}\left(\left[r_{0}, R\right] ; \mathbb{H}^{1}(\mathcal{D})\right) .
$$

Proof. Let $u \in C^{1}\left(\left[r_{0}, R\right] ; \mathbb{H}^{1}(\mathcal{D})\right)$ be a solution of (1.14) and $r \in\left[r_{0}, R\right]$. Since the variable $r$ does not intervene in the computation we can set $\phi=u(r)$ in (1.14). Then, take real parts and use that $A$ is a family of real, symmetric matrices, to obtain

$$
\begin{aligned}
\frac{1}{2} \frac{d}{d r}\|u(r)\|^{2}= & -\operatorname{Re}\left[\left\langle\lambda_{\mathrm{BC}}(r) u(r), u(r)\right\rangle_{\Gamma_{\mathrm{R}}}\right]+\operatorname{Re}[(b(r) \nabla u(r), u(r))] \\
& -(\operatorname{Im}(\beta(r)) u(r), u(r))+\operatorname{Re}(f(r), u(r)) .
\end{aligned}
$$

Since $b(r)$ is a real, vector function, by integration by parts we get

$$
\operatorname{Re}[(b(r) \nabla u(r), u(r))]=-\frac{1}{2}(\operatorname{div}(b(r)) u(r), u(r))+\frac{1}{2}\left\langle b_{1}(r) u(r), u(r)\right\rangle_{\Gamma_{\mathrm{R}}} .
$$

Combining (1.16) and (1.17) and using the condition (1.9) and the Cauchy-Schwarz inequality, we have

$$
\frac{d}{d r}\|u(r)\|^{2} \leq C\|u(r)\|^{2}+2\|f(r)\|\|u(r)\| .
$$

Integrating both sides of (1.18) with respect to $r$ we get

$$
\|u(\tau)\|^{2} \leq\left\|u\left(r_{0}\right)\right\|^{2}+\int_{r_{0}}^{\tau}\|u(s)\|[C\|u(s)\|+2\|f(s)\|] d s \quad \forall \tau \in\left[r_{0}, R\right],
$$

which yields

$$
\begin{aligned}
\max _{\tau \in\left[r_{0}, r\right]}\|u(\tau)\|^{2} & \leq\left\|u\left(r_{0}\right)\right\|^{2}+\int_{r_{0}}^{r}\|u(s)\|[C\|u(s)\|+2\|f(s)\|] d s \\
& \leq \max _{\tau \in\left[r_{0}, r\right]}\|u(\tau)\|\left(\left\|u\left(r_{0}\right)\right\|+\int_{r_{0}}^{r}[C\|u(s)\|+2\|f(s)\|] d s\right)
\end{aligned}
$$

for $r \in\left[r_{0}, R\right]$. The latter inequality obviously yields that

$$
\|u(r)\| \leq\left\|u\left(r_{0}\right)\right\|+\int_{r_{0}}^{r}[C\|u(s)\|+2\|f(s)\|] d s \quad \forall r \in\left[r_{0}, R\right],
$$

from which, after applying the Gronwall lemma, we obtain the following stability estimate:

$$
\|u(r)\| \leq C\left[\left\|u_{0}\right\|+\int_{r_{0}}^{r}\|f(s)\| d s\right] \quad \forall r \in\left[r_{0}, R\right] .
$$

From (1.19) we arrive at the uniqueness of the solution $u$ since (1.14) has a linear homogeneous part.

1.6. Finite element spaces. Let $m \in \mathbb{N}$ and $S_{h}^{m}$ be a finite-dimensional subspace of $C(\overline{\mathcal{D}}) \cap \mathbb{H}^{1}(\mathcal{D})$ consisting of complex valued functions that are polynomials of degree less than or equal to $m$ in each element (e.g. triangle or rectangle) of a nonuniform partition of $\overline{\mathcal{D}}$ with maximum diameter $h \in\left(0, h_{\star}\right]$. It is well-known, [12], that the following approximation property holds:

$$
\inf _{\chi \in S_{h}^{m}}\|v-\chi\|_{1} \leq C h^{\ell}\|v\|_{\ell+1}, \quad \ell=1, \ldots, m, \quad \forall v \in \mathbb{H}^{m+1}(\mathcal{D}), \quad \forall h \in\left(0, h_{\star}\right] .
$$

Also, we assume that the following inverse inequality holds:

$$
\|\chi\|_{1} \leq C h^{-1}\|\chi\| \quad \forall \chi \in S_{h}^{m}, \quad \forall h \in\left(0, h_{\star}\right],
$$

which is valid when, for example, the partition of $\overline{\mathcal{D}}$ is quasi-uniform (see e.g. 12]). 
1.7. Crank-Nicolson finite element approximations. For $N \in \mathbb{N}$, we consider a uniform partition of $\left[r_{0}, R\right]$ with meshlength $k:=\frac{R-r_{0}}{N}$, nodes $\left(r^{n}\right)_{n=0}^{N}$ given by $r^{n}:=n k$ for $n=0, \ldots, N$, and intermediate nodes $\left(r^{n+\frac{1}{2}}\right)_{n=0}^{N-1}$ defined by $r^{n+\frac{1}{2}}:=$ $\frac{r^{n}+r^{n+1}}{2}$ for $n=0, \ldots, N-1$. Also, for given $M \in \mathbb{N}$ and $\left(V^{m}\right)_{m=0}^{M} \subset L^{2}(\mathcal{D})$ we define $\partial_{k} V^{m}:=\frac{V^{m+1}-V^{m}}{k}$ and $\mathcal{A} V^{m}:=\frac{V^{m+1}+V^{m}}{2}$ for $m=0, \ldots, M-1$.

For $\ell=0, \ldots, N$, the numerical method derives an approximation $U^{\ell}(\cdot) \in S_{h}^{m}$ of $u\left(r^{\ell}, \cdot\right)$ as follows:

Step 1. Set

$$
U^{0}:=u_{0, h},
$$

where $u_{0, h} \in S_{h}^{m}$ is an approximation of the initial value $u_{0}$ which will be specified later.

Step 2. For $n=0, \ldots, N-1$, find $U^{n+1} \in S_{h}^{m}$ such that

$\left(\partial_{k} U^{n}, \chi\right)=-\mathrm{i} \mathcal{G}\left(r^{n+\frac{1}{2}} ; \mathcal{A} U^{n}, \chi\right)+\mathrm{i}\left(\beta\left(r^{n+\frac{1}{2}}\right) \mathcal{A} U^{n}, \chi\right)+\left(f\left(r^{n+\frac{1}{2}}\right), \chi\right) \quad \forall \chi \in S_{h}^{m}$.

1.8. An overview of the paper. In the paper at hand, we focus on the convergence analysis of the Crank-Nicolson finite element method defined by (1.22)-(1.23), proving in Section 3 an optimal order error estimate in the $L^{2}(\mathcal{D})$ norm. The convergence analysis is facing technical difficulties because the sesquilinear form $\mathcal{G}$ is (in general) not Hermitian with evolutionary dependent coefficients and the differential operator in (1.8) is of Schrödinger-type resulting in the lack of strong stability results available in parabolic problems. The authors are not aware of any scientific work analyzing finite element approximations of the solution to a multidimensional ibvp for a Schrödinger-type equation with mixed boundary conditions and coefficients depending on the evolutionary variable.

Let us give an overview of the contents of the paper.

In Section 2 we define an elliptic projection operator based on a modification $\mathcal{B}$ of the sesquilinear form $\mathcal{G}$ and provide some a priori bounds for it in Lemma 2.1. Then, in Section 2.1 we analyze its approximation properties proving optimal order error estimates in the $H^{1}(\mathcal{D})$ and the $L^{2}(\mathcal{D})$ norm, which are accomplished by using an elliptic regularity result for two-dimensional complex boundary value problems with mixed Dirichlet and Robin boundary conditions proved in Section 5. The analysis is technical since to prove an optimal order error estimate in the $L^{2}(\mathcal{D})$ norm for the evolution derivative of the error we, first, derive an optimal order error estimate on $\Gamma_{\mathrm{R}}$ for the elliptic projection error in the $|\cdot|_{-\frac{1}{2}, \Gamma_{\mathrm{R}}}$ norm.

In Section 3, we focus on the Crank-Nicolson finite element method defined in Section 1.7. First we discuss its well-posedness and stability, and then analyze its convergence in the $L^{2}(\mathcal{D})$ norm. Even though the numerical method is a combination of standard techniques to discretize an evolution problem with respect to the space and evolution variable, the error analysis developed in this section is nonstandard. This is due mainly to the fact that the sesquilinear form and the elliptic projection are $r$-dependent and calculated at the mid-points of a uniform range partition. To derive an optimal error estimate in the $L^{2}(\mathcal{D})$ norm first we define properly auxiliary test functions involving elliptic projections of the solution and its range derivatives (see (3.5)) and then use the projection estimates of Section 2 ,

In Section 4 we report on the results of some numerical experiments performed with our method, verifying experimentally the optimal order of convergence. 
A general complex elliptic boundary value problem posed on a two-dimensional rectangular domain with mixed boundary conditions is analyzed in the independent Section 5. If Dirichlet or Neumann conditions hold along the boundary, then in the weak formulation of the boundary value problem the trace integral terms vanish. A general approach of proving global regularity, [15], is to prove this estimate for half-balls, and then by change of variables, stretch the compact boundary locally and cover it by a finite union of half-balls. In our case, we analyze a complex elliptic problem posed on a rectangular domain of $\mathbb{R}^{2}$. The boundary is compact and consists of four linear segments along which Dirichlet and Robin conditions are imposed. We apply directly on this domain the half-balls technique without change of variables, as the boundary is already stretched locally. Further, we define appropriate test functions, in order to eliminate the trace terms from the weak formulation of the problem and prove the regularity estimate in Theorem 5.1 . The result is extended in Theorem 5.3. Our proof covers a class of Robin conditions related to the coefficients of the pde of the boundary value problem, a special case of which is the bottom boundary condition in (1.8).

Let us close the introduction by exposing some related bibliography. In [18, Sturm proposes and analyzes a finite element method for equation (1.1) using the boundary condition (1.5) as an interface condition over a variable bottom in the case of a multilayered fluid medium. We refer to [6] for the formulation and the convergence analysis of a Crank-Nicolson finite element method to approximate the solution to the ibvp (1.1)-(1.4). Also, we refer to [5] and [8] for the construction and the convergence analysis of a Crank-Nicolson finite difference or finite element method for the ibvp (1.1)-(1.4) and the ibvp (1.1)-(1.3), (1.5) in the axial symmetric case, proving optimal order error estimates in various norms. The approximation of the solution to problem (1.1) with Dirichlet boundary conditions over a sloping bottom has been considered in [3, 10,22. In particular, the authors of [3,22, work with the axial symmetric case constructing and analyzing Crank-Nicolson finite difference methods providing optimal order error estimates. The authors of 10 ] consider a linear Schrödinger equation in noncylindrical domains of $\mathbb{R}^{d}$ (with $d \in \mathbb{N}$ ) formulating a discontinuous Galerkin method for which a sharp error estimate is provided. The wide-angle parabolic equation consists of an alternative approximate model for the Helmholtz equation in underwater acoustics, the investigation of which over sloping domains faces the challenging question of what is the correct set of boundary conditions that one has to impose on the bottom, given its physical properties (see e.g. 4, 7, 9, 13,).

\section{An ELLIPTIC PROJECTION}

Let $\delta \geq 1+\frac{1}{2 C_{\star}}\left[\sup _{\left[r_{0}, R\right] \times \mathcal{D}}|b|+C_{2, \mathrm{TR}} \sup _{\left[r_{0}, R\right] \times\left[\theta_{A}, \theta_{B}\right]}\left|\operatorname{Im}\left(\lambda_{\mathrm{BC}}\right)\right|\right]^{2}$, where $C_{\star}$ is the constant in (1.10) and $C_{2, \mathrm{TR}}$ the constant in (1.13). Then, for $r \in\left[r_{0}, R\right]$, we define an auxiliary sesquilinear form $\mathcal{B}(r ; \cdot, \cdot): H^{1}(\mathcal{D}) \times H^{1}(\mathcal{D}) \rightarrow \mathbb{C}$ by

$$
\mathcal{B}(r ; v, w):=\mathcal{G}(r ; v, w)+\delta(v, w) \quad \forall v, w \in H^{1}(\mathcal{D}),
$$

where $\mathcal{G}(r ; \cdot, \cdot)$ is the sesquilinear form defined in (1.15). Using the trace inequality (1.12) and the Cauchy-Schwarz inequality we obtain

$$
|\mathcal{B}(r ; v, w)| \leq C_{B}\|v\|_{1}\|w\|_{1} \quad \forall v, w \in H^{1}(\mathcal{D}), \quad \forall r \in\left[r_{0}, R\right],
$$


with $C_{B}=\max \left\{\delta, \sup _{\left[r_{0}, R\right] \times \mathcal{D}}|b|, C_{1, \mathrm{TR}} \sup _{\left[r_{0}, R\right] \times\left[\theta_{A}, \theta_{B}\right]}\left|\lambda_{\mathrm{BC}}\right|, \sup _{\left[r_{0}, R\right] \times \mathcal{D}}|A|_{2}\right\}$, where $|\cdot|_{2}$ is the Euclidean matrix norm and $C_{1, \mathrm{TR}}$ is the constant in (1.12). Also, using (1.13), (1.10) and the Cauchy-Schwarz inequality, we conclude that

$$
\operatorname{Re}[\mathcal{B}(r ; v, v)] \geq C_{E}\|v\|_{1}^{2} \quad \forall v \in \mathbb{H}^{1}(\mathcal{D}), \quad \forall r \in\left[r_{0}, R\right],
$$

where $C_{E}=\min \left\{\frac{C_{\star}}{2}, 1\right\}$. Now, for $r \in\left[r_{0}, R\right]$, we define an elliptic (or a Ritz) projection $R_{h}(r): H^{1}(\mathcal{D}) \rightarrow S_{h}^{m}$ by

$$
\mathcal{B}\left(r ; R_{h}(r) v, \chi\right)=\mathcal{B}(r ; v, \chi) \quad \forall \chi \in S_{h}^{m},
$$

which is well-defined due to the coercivity property (2.3). In the following lemma we provide some a priori bounds for the elliptic projection.

Lemma 2.1. Let $\ell \in \mathbb{N}_{0}$. Then, there exists a positive constant $C_{\ell}$ such that

$$
\left\|\partial_{r}^{\ell}\left(R_{h}(r) g(r)\right)\right\|_{1} \leq C_{\ell} \sum_{j=0}^{\ell}\left\|\partial_{r}^{j} g(r)\right\|_{1}
$$

for $g \in C^{\ell}\left(\left[r_{0}, R\right] ; H^{1}(\mathcal{D})\right), h \in\left(0, h_{\star}\right]$ and $r \in\left[r_{0}, R\right]$.

Proof. The proof follows an induction argument with respect to $\ell$. Also, to simplify the notation we set $\rho(r):=R_{h}(r) g(r)$ for $r \in\left[r_{0}, R\right]$.

First, set $\chi=\rho(r)$ in (2.4) and then use (2.3) and (2.2) to get

$$
C_{E}\|\rho(r)\|_{1}^{2} \leq C_{B}\|g(r)\|_{1}\|\rho(r)\|_{1},
$$

which yields (2.5) for $\ell=0$. Now, we assume that (2.5) holds for $\ell=0, \ldots, \nu-1$, where $\nu \in \mathbb{N}$. Taking the $\partial_{r}^{\nu}$ derivative of both sides of (2.4) we get

$$
\begin{aligned}
\mathcal{B}\left(r ; \partial_{r}^{\nu} \rho(r), \chi\right)=-\sum_{j=0}^{\nu-1}\left(\begin{array}{l}
\nu \\
j
\end{array}\right) & \partial_{r}^{\nu-j} \mathcal{B}\left(r ; \partial_{r}^{j} \rho(r), \chi\right) \\
& +\sum_{j=0}^{\nu}\left(\begin{array}{c}
\nu \\
j
\end{array}\right) \partial_{r}^{\nu-j} \mathcal{B}\left(r ; \partial_{r}^{j} g(r), \chi\right) \quad \forall \chi \in S_{h}^{m},
\end{aligned}
$$

where the sesquilinear form $\partial_{r}^{\nu-j} \mathcal{B}(r ; \cdot, \cdot): H^{1}(\mathcal{D}) \times H^{1}(\mathcal{D}) \rightarrow \mathbb{C}$ follows from the sesquilinear form $\mathcal{B}$ by differentiating the coefficients with respect to $r$. Next, we set $\chi=\partial_{r}^{\nu} \rho(r)$ in (2.6) and use (2.3) and (2.2), to obtain

$$
\left\|\partial_{r}^{\nu} \rho(r)\right\|_{1} \leq C\left(\sum_{j=0}^{\nu-1}\left\|\partial_{r}^{j} \rho(r)\right\|_{1}+\sum_{j=0}^{\nu}\left\|\partial_{r}^{j} g(r)\right\|_{1}\right) .
$$

Combining (2.7) and the induction assumption we arrive at (2.5) with $\ell=\nu$.

Remark 2.2. If (1.9) holds as an equality (see Remark 1.4), then the sesquilinear form $\mathcal{B}$ is Hermitian. Therefore, if $f=0$, setting $\phi=\partial_{r} u(r)-\mathrm{i}(\beta(r)+\delta) u(r)$ in (1.14) and then taking imaginary parts and using (2.3) we obtain

$$
\frac{1}{2} \frac{d}{d r} \operatorname{Re}[\mathcal{B}(r ; u(r), u(r))] \leq c\|u(r)\|_{1}^{2} \leq c \operatorname{Re}[\mathcal{B}(r ; u(r), u(r))]
$$

for $r \in\left[r_{0}, R\right]$, which, after the application of the Gronwall lemma, yields that $\operatorname{Re}[\mathcal{B}(r ; u(r), u(r))] \leq c \operatorname{Re}\left[\mathcal{B}\left(0 ; u_{0}, u_{0}\right)\right]$ for $r \in[0, R]$. Combining the latter equation with (2.3) and (2.2), we arrive at an $H^{1}$ stability estimate. 
2.1. Approximation estimates. In this section we analyze some approximation properties of the elliptic projection defined in (2.4), which we will use often in the error analysis of Section 3 . The analysis below is based on the techniques proposed in 20] and 14 .

Let $r \in\left[r_{0}, R\right]$. First, we define an elliptic differential operator $\mathcal{L}^{*}(r): H^{2}(\mathcal{D}) \rightarrow$ $L^{2}(\mathcal{D})$ by

$$
\mathcal{L}^{*}(r) w:=-\operatorname{div}(A(r, \cdot) \nabla w)+\mathrm{i} b(r, \cdot) \nabla w+\mathrm{i} \operatorname{div}(b(r, \cdot)) w+\delta w \quad \forall w \in H^{2}(\mathcal{D}) .
$$

Then, we introduce an operator $T^{*}(r): L^{2}(\mathcal{D}) \rightarrow \mathbb{H}^{2}(\mathcal{D})$ as the solution operator of the following elliptic boundary value problem: for given $g \in L^{2}(\mathcal{D})$, find $w(r) \in$ $\mathbb{H}^{2}(\mathcal{D})$ such that

$$
\begin{aligned}
& \mathcal{L}^{*}(r) w(r)=g, \\
& \mathbf{n}^{T} A(r, y, \theta) \nabla w(r, y, \theta)=\mathrm{i} \mu_{\mathrm{BC}}(r, \theta) w(r, y, \theta) \quad \forall(y, \theta) \in \Gamma_{\mathrm{R}},
\end{aligned}
$$

where $\mu_{\mathrm{BC}}:\left[r_{0}, R\right] \times\left[\theta_{A}, \theta_{B}\right] \rightarrow \mathbb{C}$ is defined by $\mu_{\mathrm{BC}}(r, \theta):=b_{1}(r, 1, \theta)-\overline{\lambda_{\mathrm{BC}}}(r, \theta)$ for $r \in\left[r_{0}, R\right]$ and $\theta \in\left[\theta_{A}, \theta_{B}\right]$. The operator $T^{*}(r)$ is well-defined since the elliptic boundary value problem (2.8) has unique weak solution $T^{*}(r) g \in \mathbb{H}^{1}(\mathcal{D})$ which, according to the elliptic regularity result of Section 5 , also belongs to $\mathbb{H}^{2}(\mathcal{D})$ and has the following property: there exists a constant $C>0$ that depends on $\left[r_{0}, R\right]$ and $\mathcal{D}$ such that

$$
\left\|T^{*}(r) g\right\|_{2} \leq C\|g\| .
$$

Remark 2.3. We note that if $w_{1} \in \mathbb{H}^{2}(\mathcal{D})$ and satisfies the Robin-type boundary condition in (2.8), then it holds that

$$
\left(\mathcal{L}^{*}(r) w_{1}, w_{2}\right)=\overline{\mathcal{B}\left(r ; w_{2}, w_{1}\right)} \quad \forall w_{2} \in \mathbb{H}^{2}(\mathcal{D}) .
$$

Proposition 2.4. There exists a positive constant $C$ such that

$$
\left\|R_{h}(r) v-v\right\|+h\left\|R_{h}(r) v-v\right\|_{1} \leq C h^{m+1}\|v\|_{m+1}
$$

for $v \in \mathbb{H}^{m+1}(\mathcal{D}), h \in\left(0, h_{\star}\right]$ and $r \in\left[r_{0}, R\right]$.

Proof. Let $e(r):=R_{h}(r) v-v$. Using (2.4), (2.3) and (2.2), we obtain

$$
\begin{aligned}
C_{E}\|e(r)\|_{1}^{2} & \leq \operatorname{Re}[\mathcal{B}(r ; e(r), e(r))] \\
& \leq \operatorname{Re}[\mathcal{B}(r ; e(r), \chi-v)] \\
& \leq C\|e(r)\|_{1}\|\chi-v\|_{1} \quad \forall \chi \in S_{h}^{m},
\end{aligned}
$$

which, along with (1.20), yields

$$
\begin{aligned}
\|e(r)\|_{1} & \leq C \inf _{\chi \in S_{h}^{m}}\|v-\chi\|_{1} \\
& \leq C h^{m}\|v\|_{m+1} .
\end{aligned}
$$

Now, let $w(r):=T^{*}(r) e(r)$. Then, using (2.10), (2.4), (2.2) and (2.12), we obtain

$$
\begin{aligned}
\|e(r)\|^{2} & =\left(\mathcal{L}^{*}(r) w(r), e(r)\right) \\
& =\overline{\mathcal{B}(r ; e(r), w(r)-\chi)} \\
& \leq C h^{m}\|w(r)-\chi\|_{1}\|v\|_{m+1} \quad \forall \chi \in S_{h}^{m+1} .
\end{aligned}
$$


Now, we use (1.20) and (2.9) to get

$$
\begin{aligned}
\inf _{\chi \in S_{h}^{m+1}}\|w(r)-\chi\|_{1} & \leq C h\|w(r)\|_{2} \\
& \leq C h\|e(r)\| .
\end{aligned}
$$

Combining (2.13) and (2.14) we obtain

$$
\|e(r)\| \leq C h^{m+1}\|v\|_{m+1} .
$$

Finally, (2.11) follows as a simple consequence of (2.13) and (2.15).

Proposition 2.5. For $\ell \in \mathbb{N}$, there exists a positive constant $C_{\ell}$ such that

$$
\left\|\partial_{r}^{\ell}\left(R_{h}(r) g(r)-g(r)\right)\right\|_{1} \leq C_{\ell} h^{m} \sum_{j=0}^{\ell}\left\|\partial_{r}^{j} g(r)\right\|_{m+1}
$$

for $g \in C^{\ell}\left(\left[r_{0}, R\right] ; \mathbb{H}^{m+1}(\mathcal{D})\right), h \in\left(0, h_{\star}\right]$ and $r \in\left[r_{0}, R\right]$.

Proof. We will get the error bound (2.16) by induction with respect to $\ell$.

Let $e(r):=R_{h}(r) g(r)-g(r)$ and $\nu \in \mathbb{N}$. Then, differentiating with respect to $r$ both sides of (2.4) we obtain

$$
\mathcal{B}\left(r ; \partial_{r}^{\nu} e(r), \chi\right)=-\sum_{j=0}^{\nu-1}\left(\begin{array}{l}
\nu \\
j
\end{array}\right) \partial_{r}^{\nu-j} \mathcal{B}\left(r ; \partial_{r}^{j} e(r), \chi\right) \quad \forall \chi \in S_{h}^{m}
$$

Let us assume that (2.16) holds for $\ell=0, \ldots, \nu-1$, which is true when $\nu=1$ due to (2.11). Now, using (2.3), (2.17) and (2.2), we have

$$
\begin{aligned}
C_{E}\left\|\partial_{r}^{\nu} e(r)\right\|_{1}^{2} \leq & \mathcal{B}\left(r ; \partial_{r}^{\nu} e(r), \partial_{r}^{\nu} e(r)\right) \\
\leq & \mathcal{B}\left(r ; \partial_{r}^{\nu} e(r), \partial_{r}^{\nu} e(r)+\chi\right)-\mathcal{B}\left(r ; \partial_{r}^{\nu} e(r), \chi\right) \\
\leq & \mathcal{B}\left(r ; \partial_{r}^{\nu} e(r), \partial_{r}^{\nu} e(r)+\chi\right)+\sum_{j=0}^{\nu-1}\left(\begin{array}{c}
\nu \\
j
\end{array}\right) \partial_{r}^{\nu-j} \mathcal{B}\left(r ; \partial_{r}^{j} e(r), \chi\right) \\
\leq & C\left[\left\|\partial_{r}^{\nu} e(r)\right\|_{1}\left\|\partial_{r}^{\nu} e(r)+\chi\right\|_{1}+\|\chi\|_{1} \sum_{j=0}^{\nu-1}\left\|\partial_{r}^{j} e(r)\right\|_{1}\right] \\
\leq & C\left[\left\|\partial_{r}^{\nu} e(r)\right\|_{1}\left\|\partial_{r}^{\nu} e(r)+\chi\right\|_{1}\right. \\
& \left.+\left(\left\|\partial_{r}^{\nu} e(r)+\chi\right\|_{1}+\left\|\partial_{r}^{\nu} e(r)\right\|_{1}\right) \sum_{j=0}^{\nu-1}\left\|\partial_{r}^{j} e(r)\right\|_{1}\right] \\
\leq & C\left[\left(\sum_{j=0}^{\nu}\left\|\partial_{r}^{j} e(r)\right\|_{1}\right)\left\|\partial_{r}^{\nu} e(r)+\chi\right\|_{1}+\left\|\partial_{r}^{\nu} e(r)\right\|_{1} \sum_{j=0}^{\nu-1}\left\|\partial_{r}^{j} e(r)\right\|_{1}\right]
\end{aligned}
$$


for $\chi \in S_{h}^{m}$, which yields

$$
\begin{aligned}
&\left\|\partial_{r}^{\nu} e(r)\right\|_{1}^{2} \leq C {\left[\left(\sum_{j=0}^{\nu}\left\|\partial_{r}^{j} e(r)\right\|_{1}\right) \inf _{\chi \in S_{h}^{m}}\left\|\partial_{r}^{\nu} g(r)-\chi\right\|_{1}+\left\|\partial_{r}^{\nu} e(r)\right\|_{1} \sum_{j=0}^{\nu-1}\left\|\partial_{r}^{j} e(r)\right\|_{1}\right] } \\
& \leq C\left\|\partial_{r}^{\nu} e(r)\right\|_{1}\left[\sum_{j=0}^{\nu-1}\left\|\partial_{r}^{j} e(r)\right\|_{1}+\inf _{\chi \in S_{h}^{m}}\left\|\partial_{r}^{\nu} g(r)-\chi\right\|_{1}\right] \\
&+C \inf _{\chi \in S_{h}^{m}}\left\|\partial_{r}^{\nu} g(r)-\chi\right\|_{1} \sum_{j=0}^{\nu-1}\left\|\partial_{r}^{j} e(r)\right\|_{1} .
\end{aligned}
$$

Next, using the arithmetic mean inequality, it follows that

$$
\begin{array}{r}
\left\|\partial_{r}^{\nu} e(r)\right\|_{1}^{2} \leq C\left\{\left[\sum_{j=0}^{\nu-1}\left\|\partial_{r}^{j} e(r)\right\|_{1}+\inf _{\chi \in S_{h}^{m}}\left\|\partial_{r}^{\nu} g(r)-\chi\right\|_{1}\right]^{2}\right. \\
\left.+\inf _{\chi \in S_{h}^{m}}\left\|\partial_{r}^{\nu} g(r)-\chi\right\|_{1} \sum_{j=0}^{\nu-1}\left\|\partial_{r}^{j} e(r)\right\|_{1}\right\} \\
\leq C\left[\left(\sum_{j=0}^{\nu-1}\left\|\partial_{r}^{j} e(r)\right\|_{1}\right)^{2}+\inf _{\chi \in S_{h}^{m}}\left\|\partial_{r}^{\nu} g(r)-\chi\right\|_{1}^{2}\right] .
\end{array}
$$

Finally, using the induction assumption and (1.20), from the inequality above we obtain

$$
\left\|\partial_{r}^{\nu} e(r)\right\|_{1}^{2} \leq C h^{2 m}\left[\left(\sum_{j=0}^{\nu-1}\left\|\partial_{r}^{j} g(r)\right\|_{m+1}\right)^{2}+\left\|\partial_{r}^{\nu} g(r)\right\|_{m+1}^{2}\right]
$$

which yields (2.16) for $\ell=\nu$.

Using a technique introduced in [14, we are able to derive an optimal order estimate of the elliptic projection error in the $|\cdot|_{-\frac{1}{2}, \Gamma_{\mathrm{R}}}$ norm which allows us to obtain the following optimal order approximation result for the time-derivative of the elliptic projection.

Proposition 2.6. There exists a positive constant $C$ such that

$$
\left\|\partial_{r}\left(R_{h}(r) g(r)-g(r)\right)\right\| \leq C h^{m+1}\left(\|g(r)\|_{m+1}+\left\|\partial_{r} g(r)\right\|_{m+1}\right)
$$

for $g \in C^{1}\left(\left[r_{0}, R\right] ; \mathbb{H}^{m+1}(\mathcal{D})\right), h \in\left(0, h_{\star}\right]$ and $r \in\left[r_{0}, R\right]$. 
Proof. Let $e(r):=R_{h}(r) g(r)-g(r)$ and $w(r)=T^{*}(r) \partial_{r} e(r)$. Then, using (2.10) and (2.17), we have

$$
\begin{aligned}
\left\|\partial_{r} e(r)\right\|^{2}= & \operatorname{Re}\left[\left(\mathcal{L}^{*}(r) w(r), \partial_{r} e(r)\right)\right] \\
= & \operatorname{Re}\left[\mathcal{B}\left(r ; \partial_{r} e(r), w(r)\right)\right] \\
= & \operatorname{Re}\left[\mathcal{B}\left(r ; \partial_{r} e(r), w(r)-\chi\right)-\partial_{r} \mathcal{B}(r ; e(r), \chi)\right] \\
= & \operatorname{Re}\left[\mathcal{B}\left(r ; \partial_{r} e(r), w(r)-\chi\right)-\partial_{r} \mathcal{B}(r ; e(r), \chi-w(r))\right. \\
\leq & \left.\quad-\partial_{r} \mathcal{B}(r ; e(r), w(r))\right] \\
\leq & \left(\left\|\partial_{r} e(r)\right\|_{1}+\|e(r)\|_{1}\right)\|w(r)-\chi\|_{1} \\
& -\operatorname{Re}\left[\partial_{r} \mathcal{B}(r ; e(r), w(r))\right]
\end{aligned}
$$

for $\chi \in S_{h}^{m}$. Setting $I(r):=-\operatorname{Re}\left[\partial_{r} \mathcal{B}(r ; e(r), w(r))\right]$ and using (2.11) and (2.16), (2.19) yields

$$
\begin{aligned}
\left\|\partial_{r} e(r)\right\|^{2} & \leq C\left(\left\|\partial_{r} e(r)\right\|_{1}+\|e(r)\|\right) \inf _{\chi \in S_{h}^{m}}\|w(r)-\chi\|_{1}+I(r) \\
& \leq C h^{m+1}\left[\|g(r)\|_{m+1}+\left\|\partial_{r} g(r)\right\|_{m+1}\right]\|w(r)\|_{2}+I(r) .
\end{aligned}
$$

First, we observe that

$$
I(r)=\sum_{j=1}^{3} I_{j}(r),
$$

where

$$
\begin{gathered}
I_{1}(r):=-\operatorname{Re}\left[\mathrm{i}\left(\partial_{r} b(r) \nabla e(r), w(r)\right)\right], \quad I_{2}(r):=-\operatorname{Re}\left[-\mathrm{i}\left\langle\partial_{r} \lambda_{\mathrm{BC}}(r) e(r), w(r)\right\rangle_{\Gamma_{\mathrm{R}}}\right], \\
I_{3}(r):=-\operatorname{Re}\left[\left(\partial_{r} A(r) \nabla e(r), \nabla w(r)\right)\right] .
\end{gathered}
$$

Now, using integration by parts we obtain

$$
\begin{gathered}
I_{1}(r)=-\operatorname{Re}\left[-\mathrm{i}\left(\operatorname{div}\left(\partial_{r} b(r)\right) e(r), w(r)\right)-\mathrm{i}\left(\partial_{r} b(r) e(r), \nabla w(r)\right)\right. \\
\left.+\mathrm{i}\left\langle\partial_{r} b_{1}(r) e(r), w(r)\right\rangle_{\Gamma_{\mathrm{R}}}\right] \\
\leq C\|e(r)\|\|w(r)\|_{1}-\operatorname{Re}\left[\mathrm{i}\left\langle\partial_{r} b_{1}(r) e(r), w(r)\right\rangle_{\Gamma_{\mathrm{R}}}\right] .
\end{gathered}
$$

Next, we use (2.22) and (1.11), to obtain

$$
\begin{gathered}
I_{1}(r)+I_{2}(r) \leq-\operatorname{Re}\left[-\mathrm{i}\left\langle\left(\partial_{r} \lambda_{\mathrm{G}}(r)-\partial_{r} b_{1}(r)\right) e(r), w(r)\right\rangle_{\Gamma_{\mathrm{R}}}\right] \\
+C\|e(r)\|\|w(r)\|_{1} \\
\leq C\left[|e(r)|_{-\frac{1}{2}, \Gamma_{\mathrm{R}}}+\|e(r)\|\right]\|w(r)\|_{1} .
\end{gathered}
$$

In addition, using integration by parts, we have

$$
\begin{aligned}
I_{3}(r) & =-\operatorname{Re}\left[-\left(\operatorname{div}\left(\partial_{r}(A(r) \nabla w(r))\right), e(r)\right)+\left\langle\mathbf{n}^{T} \partial_{r} A(r) \nabla w(r), e(r)\right\rangle_{\Gamma_{\mathrm{R}}}\right] \\
& \leq C\|w(r)\|_{2}\|e(r)\|+\operatorname{Re}\left[\left\langle\mathbf{n}^{T} \partial_{r} A(r) \nabla w(r), e(r)\right\rangle_{\Gamma_{\mathrm{R}}}\right] \\
& \leq C\left[\|e(r)\|+|e(r)|_{-\frac{1}{2}, \Gamma_{\mathrm{R}}}\right]\|w(r)\|_{2} .
\end{aligned}
$$

Combining (2.21), (2.23) and (2.24), we arrive at

$$
I(r) \leq C\left[\|e(r)\|+|e(r)|_{-\frac{1}{2}, \Gamma_{\mathrm{R}}}\right]\|w(r)\|_{2} .
$$


For $\varepsilon \in H^{\frac{1}{2}}\left(\Gamma_{\mathrm{R}}\right)$, let $z(r) \in \mathbb{H}^{2}(\mathcal{D})$ be the solution of the following elliptic boundary value problem:

$$
\begin{aligned}
& \mathcal{L}^{*}(r) z(r)=0, \\
& \mathbf{n}^{T} A(r, y, \theta) \nabla z(r, y, \theta)=\mathrm{i} \mu_{\mathrm{BC}}(r, \theta) z(r, y, \theta)+\varepsilon(r, y, \theta) \quad \forall(y, \theta) \in \Gamma_{\mathrm{R}} .
\end{aligned}
$$

Since $\left(\mathcal{L}^{*}(r) z(r), e(r)\right)=0$, after integrating by parts and using (2.4), we get

$$
\langle e(r), \varepsilon\rangle_{\Gamma_{\mathrm{R}}}=\overline{\mathcal{B}(r ; e(r), z(r)-\chi)} \quad \forall \chi \in S_{h}^{m} .
$$

Using (2.27), (2.2), (1.20) and (2.11), we obtain

$$
\begin{aligned}
\left|\langle e(r), \varepsilon\rangle_{\Gamma_{\mathrm{R}}}\right| & \leq C\|e(r)\|_{1} \inf _{\chi \in S_{h}^{m}}\|z(r)-\chi\|_{1} \\
& \leq C h^{m+1}\|g(r)\|_{m+1}\|z(r)\|_{2} .
\end{aligned}
$$

The elliptic regularity result (cf. Theorem 5.3 and Remark 5.4) for the elliptic problem (2.26) yields the existence of a constant $C>0$ that depends on $\left[r_{0}, R\right]$ and $\mathcal{D}$ such that

$$
\|z(r)\|_{2} \leq C|\varepsilon|_{\frac{1}{2}, \Gamma_{\mathrm{R}}} .
$$

Thus, combining (2.28) and (2.29), we conclude that

$$
\begin{aligned}
\left|\left\langle e(r), \tau_{\partial \mathcal{D}} \phi\right\rangle_{\Gamma_{\mathrm{R}}}\right| & \leq\left. C h^{m+1}\|g(r)\|_{m+1}\left|\tau_{\partial \mathcal{D}} \phi\right|_{\Gamma_{\mathrm{R}}}\right|_{\frac{1}{2}, \Gamma_{\mathrm{R}}} \\
& \leq C h^{m+1}\|g(r)\|_{m+1}\|\phi\|_{1} \quad \forall \phi \in \mathbb{H}^{1}(\mathcal{D}),
\end{aligned}
$$

which yields

$$
|e(r)|_{-\frac{1}{2}, \Gamma_{\mathrm{R}}} \leq C h^{m+1}\|g(r)\|_{m+1} .
$$

Therefore, (2.25), (2.30) and (2.11) yield

$$
I(r) \leq C h^{m+1}\|g(r)\|_{m+1}\|w(r)\|_{2} .
$$

Finally, we use (2.20), (2.31) and (2.9) to have

$$
\left\|\partial_{r} e(r)\right\|^{2} \leq C h^{m+1}\left(\|g(r)\|_{m+1}+\left\|\partial_{r} g(r)\right\|_{m+1}\right)\left\|\partial_{r} e(r)\right\|,
$$

which obviously yields (2.18).

\section{Convergence Analysis}

3.1. Stability results for the numerical method. We show below that the numerical method is well-defined and stable when $k$ is small enough.

Proposition 3.1. There exists a constant $C_{0} \geq 0$ such that the Crank-Nicolson finite element method (1.23) is well-defined when $C_{0} k<1$.

Proof. Let $\phi \in S_{h}^{m}, r \in\left[r_{0}, R\right]$ and $\Lambda_{h}(\phi ; \cdot): S_{h}^{m} \rightarrow \mathbb{C}$ be defined by

$$
\Lambda_{h}(\phi ; \chi):=(\phi, \chi)+\frac{\mathrm{i} k}{2} \mathcal{G}(r ; \phi, \chi)-\frac{\mathrm{i} k}{2}(\beta(r) \phi, \chi) \quad \forall \chi \in S_{h}^{m} .
$$

It is easily seen that the numerical method (1.23) yields at every range step a linear system of algebraic equations, the matrix of which is invertible iff the assumption $\Lambda_{h}(\phi ; \cdot)=0$ implies that $\phi=0$.

Let $\phi_{\star} \in S_{h}^{m}$ such that $\Lambda_{h}\left(\phi_{\star}, \chi\right)=0$ for $\chi \in S_{h}^{m}$. Then, we have $\operatorname{Re}\left[\Lambda_{h}\left(\phi_{\star} ; \phi_{\star}\right)\right]$ $=0$ which yields that

$$
\left\|\phi_{\star}\right\|^{2}+\frac{k}{2}\left[\left\langle\operatorname{Re}\left(\lambda_{\mathrm{BC}}(r)\right) \phi_{\star}, \phi_{\star}\right\rangle_{\Gamma_{\mathrm{R}}}-\operatorname{Re}\left[\left(b(r) \nabla \phi_{\star}, \phi_{\star}\right)\right]\right]+\frac{k}{2}\left(\operatorname{Im}(\beta(r)) \phi_{\star}, \phi_{\star}\right)=0 .
$$


Using integration by parts we obtain

$$
\operatorname{Re}\left[\left(b(r) \nabla \phi_{\star}, \phi_{\star}\right)\right]=-\frac{1}{2}\left(\operatorname{div}(b(r)) \phi_{\star}, \phi_{\star}\right)+\frac{1}{2}\left\langle b_{1}(r) \phi_{\star}, \phi_{\star}\right\rangle_{\Gamma_{\mathrm{R}}} .
$$

Then, combine (3.1) and (3.2) to obtain

$\left\|\phi_{\star}\right\|^{2}+\frac{k}{4}\left\langle\left(2 \operatorname{Re}\left(\lambda_{\mathrm{BC}}(r)-b_{1}(r)\right)\right) \phi_{\star}, \phi_{\star}\right\rangle_{\Gamma_{\mathrm{R}}}=-\frac{k}{4}\left((\operatorname{Im}(2 \beta(r))+\operatorname{div}(b(r))) \phi_{\star}, \phi_{\star}\right)$

which, along with (1.9), yields $\left\|\phi_{\star}\right\|^{2}\left(1-C_{0} k\right) \leq 0$ with

$$
C_{0}=\frac{1}{4} \sup _{\left[r_{0}, R\right] \times \mathcal{D}}|2 \operatorname{Im}(\beta)+\operatorname{div}(b)| .
$$

Thus, if $1-C_{0} k>0$, then $\phi_{\star}=0$.

Remark 3.2. In the physical problem (1.7), the equality holds in (1.9) and we have $\operatorname{div}(b(r))=\frac{\partial_{r} s(r, \theta)}{s(r, \theta)}$ and $2 \operatorname{Im}(\beta(r, y, \theta))=-\frac{\partial_{r} s(r, \theta)}{s(r, \theta)}$ since $\gamma_{R F}$ is real valued. Thus, from (3.3) it follows that $\left\|\phi_{\star}\right\|^{2} \leq 0$ or $\phi_{\star}=0$, which means that the numerical method is unconditionally well-defined.

Remark 3.3. Assuming that (1.9) holds, in the proof of Proposition 3.1 we proved that the following inequality holds:

$$
\operatorname{Re}[-\mathrm{i} \mathcal{G}(r ; w, w)] \leq C\|w\|^{2} \quad \forall w \in \mathbb{H}^{1}(\mathcal{D}), \quad \forall r \in\left[r_{0}, R\right] .
$$

Proposition 3.4. Let $C_{0}$ be the nonnegative constant specified in Proposition 3.1. Then, there exists a constant $c_{\star} \geq C_{0}$ such that the Crank-Nicolson finite element method (1.23) is $L^{2}$-stable when $c_{\star} k<1$.

Proof. First, we assume that $C_{0} k<1$ in order to ensure that the numerical approximations are well-defined. Then, we set $\chi=\mathcal{A} U^{n}$ in (1.23) and take real parts to obtain

$$
\begin{array}{rl}
\left\|U^{n+1}\right\|^{2}-\left\|U^{n}\right\|^{2}=2 & k \operatorname{Re}\left[-\mathrm{i} \mathcal{G}\left(r^{n+\frac{1}{2}} ; \mathcal{A} U^{n}, \mathcal{A} U^{n}\right)\right] \\
& -2 k \operatorname{Im}\left[\left(\beta\left(r^{n+\frac{1}{2}}\right) \mathcal{A} U^{n}, \mathcal{A} U^{n}\right)\right] \\
+ & 2 k \operatorname{Re}\left[\left(f\left(r^{n+\frac{1}{2}}\right), \mathcal{A} U^{n}\right)\right], \quad n=0, \ldots, N-1 .
\end{array}
$$

Using the Cauchy-Schwarz inequality and (3.4) from the latter equation it follows that there exists $\widetilde{C}>0$ such that

$$
\left\|U^{n+1}\right\|^{2}-\left\|U^{n}\right\|^{2} \leq \widetilde{C} k\left(\left\|U^{n+1}\right\|+\left\|U^{n}\right\|\right)\left(\left\|f\left(r^{n+\frac{1}{2}}\right)\right\|+\left\|U^{n+1}\right\|+\left\|U^{n}\right\|\right)
$$

for $n=0, \ldots, N-1$. Observing that

$$
\left\|U^{n+1}\right\|^{2}-\left\|U^{n}\right\|^{2}=\left(\left\|U^{n+1}\right\|-\left\|U^{n}\right\|\right)\left(\left\|U^{n+1}\right\|+\left\|U^{n}\right\|\right),
$$

the inequality above yields

$$
(1-\widetilde{C} k)\left\|U^{n+1}\right\| \leq(1+\widetilde{C} k)\left\|U^{n}\right\|+\widetilde{C} k\left\|f\left(r^{n+\frac{1}{2}}\right)\right\|, \quad n=0, \ldots, N-1 .
$$

Thus, letting $c_{\star}=\max \left\{C_{0}, 3 \widetilde{C}\right\}$ and assuming that $c_{\star} k<1$, we arrive at

$$
\left\|U^{n+1}\right\| \leq e^{3 \widetilde{C} k}\left\|U^{n}\right\|+\frac{3 \widetilde{C}}{2} k\left\|f\left(r^{n+\frac{1}{2}}\right)\right\|, \quad n=0, \ldots, N-1,
$$


from which, after applying a standard discrete Gronwall argument, we get

$$
\left\|U^{n}\right\| \leq C\left(\left\|U^{0}\right\|+\sup _{r \in\left[r_{0}, R\right]}\|f(r)\|\right), \quad n=0, \ldots, N,
$$

which indicates the $L^{2}$ stability of the method.

3.2. Error estimates. In this section, to simplify the notation we set $\varrho(r):=$ $R_{h}(r) u(r), e(r):=u(r)-\varrho(r)$ and $\sigma(r):=R_{h}(r) \partial_{r}^{2} u(r)$ for $r \in\left[r_{0}, R\right]$, and $\varrho^{n}:=$ $\varrho\left(r^{n}\right), e^{n}:=e\left(r^{n}\right), \sigma^{n}:=\sigma\left(r^{n}\right)$ and $u^{n}:=u\left(r^{n}\right)$ for $n=0, \ldots, N$. Let us define $\left(\theta^{n}\right)_{n=0}^{N} \subset S_{h}^{m}$ by

$$
\begin{aligned}
\theta^{n} & :=U^{n}-R_{h}\left(r^{n}\right) u\left(r^{n}\right)+\frac{k^{2}}{8} R_{h}\left(r^{n}\right) \partial_{r}^{2} u\left(r^{n}\right) \\
& =U^{n}-\varrho^{n}+\frac{k^{2}}{8} \sigma^{n}, \quad n=0, \ldots, N .
\end{aligned}
$$

Using (1.23) and (1.14), we arrive at the following error equation:

$$
\begin{aligned}
& \left(\partial_{k} \theta^{n}, \chi\right)+\mathrm{i} \mathcal{B}\left(r^{n+\frac{1}{2}} ; \mathcal{A} \theta^{n}, \chi\right) \\
& \quad=\mathrm{i}\left(\left(\delta+\beta\left(r^{n+\frac{1}{2}}\right)\right) \mathcal{A} \theta^{n}, \chi\right)+\sum_{j=1}^{6} T_{j}(\chi), \quad \forall \chi \in S_{h}^{m},
\end{aligned}
$$

where

$$
\begin{aligned}
& T_{1}(\chi):=\left(\partial_{r} u\left(r^{n+\frac{1}{2}}\right)-\partial_{k} \varrho^{n}, \chi\right), \\
& T_{2}(\chi):=\mathrm{i}\left(\left(\delta+\beta\left(r^{n+\frac{1}{2}}\right)\right)\left(\mathcal{A} u^{n}-u\left(r^{n+\frac{1}{2}}\right)\right), \chi\right), \\
& T_{3}(\chi):=\frac{k^{2}}{8}\left(\partial_{k} \sigma^{n}, \chi\right), \\
& T_{4}(\chi):=\mathrm{i}\left(\left(\delta+\beta\left(r^{n+\frac{1}{2}}\right)\right)\left(\mathcal{A} \varrho^{n}-\mathcal{A} u^{n}\right), \chi\right), \\
& T_{5}(\chi):=-\mathrm{i} \frac{k^{2}}{8}\left(\left(\delta+\beta\left(r^{n+\frac{1}{2}}\right)\right) \mathcal{A} \sigma^{n}, \chi\right), \\
& T_{6}(\chi):=\mathrm{i} \mathcal{B}\left(r^{n+\frac{1}{2}} ; u\left(r^{n+\frac{1}{2}}\right)-\mathcal{A} \varrho^{n}+\frac{k^{2}}{8} \mathcal{A} \sigma^{n}, \chi\right) .
\end{aligned}
$$

Set $\chi=\mathcal{A} \theta^{n}$ in (3.6) and then take real parts and use (3.4) to obtain

$$
\left\|\theta^{n+1}\right\|^{2}-\left\|\theta^{n}\right\|^{2} \leq C k\left(\left\|\theta^{n+1}\right\|+\left\|\theta^{n}\right\|\right)^{2}+2 k \sum_{j=1}^{6} \operatorname{Re}\left(T_{j}\left(\mathcal{A} \theta^{n}\right)\right), \quad n=0, \ldots, N-1 .
$$

Our next step is to properly bound the terms in the right-hand side of (3.7). First, using the Cauchy-Schwarz inequality, the Taylor formula and (2.11), we obtain

$$
\begin{aligned}
\operatorname{Re}\left(T_{2}(\chi)\right) & \leq C\|\chi\|\left\|\mathcal{A} u^{n}-u\left(r^{n+\frac{1}{2}}\right)\right\| \\
& \leq C\|\chi\|\left\|\int_{r^{n+\frac{1}{2}}}^{r^{n+1}}\left(r^{n+1}-s\right) \partial_{r}^{2} u(s) d s+\int_{r^{n}}^{r^{n+\frac{1}{2}}}\left(s-r^{n+1}\right) \partial_{r}^{2} u(s) d s\right\| \\
& \leq C k^{2}\|\chi\| \sup _{\left[r_{0}, R\right]}\left\|\partial_{r}^{2} u\right\|, \quad \forall \chi \in S_{h}^{m}
\end{aligned}
$$


and

$$
\begin{aligned}
\operatorname{Re}\left(T_{4}(\chi)\right) & \leq C\|\chi\|\left(\left\|e^{n}\right\|+\left\|e^{n+1}\right\|\right) \\
& \leq C h^{m+1}\|\chi\| \sup _{\left[r_{0}, R\right]}\|u\|_{m+1}, \quad \forall \chi \in S_{h}^{m} .
\end{aligned}
$$

Now, we use the Cauchy-Schwarz inequality, the Taylor formula and (2.18) to get

$$
\begin{aligned}
\operatorname{Re}\left(T_{1}(\chi)\right) & \leq\|\chi\|\left[\left\|\partial_{r} u\left(r^{n+\frac{1}{2}}\right)-\partial_{k} u^{n}\right\|+\left\|\partial_{k} e^{n}\right\|\right] \\
& \leq\|\chi\|\left[\left\|\partial_{r} u\left(r^{n+\frac{1}{2}}\right)-\partial_{k} u^{n}\right\|+\frac{1}{k}\left\|\int_{r^{n}}^{r^{n+1}} \partial_{r} e(s) d s\right\|\right] \\
& \leq C\|\chi\|\left(k^{2} \sup _{\left[r_{0}, R\right]}\left\|\partial_{r}^{3} u\right\|+\sup _{\left[r_{0}, R\right]}\left\|\partial_{r} e\right\|\right) \\
& \left.\leq C\|\chi\|\left[\begin{array}{c}
k^{2} \sup _{\left[r_{0}, R\right]}\left\|\partial_{r}^{3} u\right\| \\
+h^{m+1}\left(\sup _{\left[r_{0}, R\right]}\|u\|_{m+1}+\sup _{\left[r_{0}, R\right]}\left\|\partial_{r} u\right\|_{m+1}\right.
\end{array}\right)\right]
\end{aligned}
$$

for $\chi \in S_{h}^{m}$. Next, we use the Cauchy-Schwarz inequality and (2.5) to have

$$
\begin{aligned}
\operatorname{Re}\left(T_{3}(\chi)\right) & \leq C k\|\chi\|\left\|\int_{r^{n}}^{r^{n+1}} \partial_{r} \sigma(s) d s\right\| \\
& \leq C k^{2}\|\chi\| \sup _{\left[r_{0}, R\right]}\left\|\partial_{r} \sigma\right\| \\
& \leq C k^{2}\|\chi\|\left(\sup _{\left[r_{0}, R\right]}\left\|\partial_{r}^{2} u\right\|_{1}+\sup _{\left[r_{0}, R\right]}\left\|\partial_{r}^{3} u\right\|_{1}\right), \quad \forall \chi \in S_{h}^{m},
\end{aligned}
$$

and

$$
\begin{aligned}
\operatorname{Re}\left(T_{5}(\chi)\right) & \leq C k^{2}\|\chi\|\left(\left\|\sigma^{n}\right\|+\left\|\sigma^{n+1}\right\|\right) \\
& \leq C k^{2}\|\chi\| \sup _{\left[r_{0}, R\right]}\|\sigma\| \\
& \leq C k^{2}\|\chi\| \sup _{\left[r_{0}, R\right]}\left\|\partial_{r}^{2} u\right\|_{1}, \quad \forall \chi \in S_{h}^{m} .
\end{aligned}
$$

In order to estimate the last term on the right-hand side of (3.7) we introduce the following splitting:

$$
T_{6}(\chi)=\sum_{j=1}^{3} T_{6, j}(\chi), \quad \forall \chi \in S_{h}^{m},
$$

where

$$
\begin{aligned}
& T_{6,1}(\chi):=\mathrm{i} \mathcal{B}\left(r^{n+\frac{1}{2}} ; u\left(r^{n+\frac{1}{2}}\right)-\mathcal{A} u^{n}, \chi\right), \\
& T_{6,2}(\chi):=\mathrm{i} \mathcal{B}\left(r^{n+\frac{1}{2}} ; \mathcal{A} u^{n}-\mathcal{A} \varrho^{n}, \chi\right), \\
& T_{6,3}(\chi):=\mathrm{i} \frac{k^{2}}{8} \mathcal{B}\left(r^{n+\frac{1}{2}} ; \mathcal{A} \sigma^{n}, \chi\right) .
\end{aligned}
$$


Using (2.2), the Taylor formula and (1.21), we obtain

$$
\begin{aligned}
\operatorname{Re}\left(T_{6,1}(\chi)\right) \leq \operatorname{Re} & {\left[-\mathrm{i} \frac{k^{2}}{8} \mathcal{B}\left(r^{n+\frac{1}{2}} ; \partial_{r}^{2} u\left(r^{n+\frac{1}{2}}\right), \chi\right)\right]+C k^{4}\|\chi\|_{1} \sup _{\left[r_{0}, R\right]}\left\|\partial_{r}^{4} u\right\|_{1} } \\
\leq \operatorname{Re} & {\left[-\mathrm{i} \frac{k^{2}}{8} \mathcal{B}\left(r^{n+\frac{1}{2}} ; \partial_{r}^{2} u\left(r^{n+\frac{1}{2}}\right), \chi\right)\right] } \\
& +C k^{4} h^{-1}\|\chi\| \sup _{\left[r_{0}, R\right]}\left\|\partial_{r}^{4} u\right\|_{1}, \quad \forall \chi \in S_{h}^{m} .
\end{aligned}
$$

Now, applying the Taylor formula, (2.2), (2.4), (1.21) and (2.16), we have

$$
\begin{aligned}
\operatorname{Re}\left(T_{6,2}(\chi)\right) & =\operatorname{Re}\left[\mathrm{i} \mathcal{B}\left(r^{n+\frac{1}{2}} ; \mathcal{A} e^{n}, \chi\right)\right] \\
& \leq \operatorname{Re}\left[\mathrm{i} \mathcal{B}\left(r^{n+\frac{1}{2}} ; e\left(r^{n+\frac{1}{2}}\right), \chi\right)\right]+C k^{2} \sup _{\left[r_{0}, R\right]}\left\|\partial_{r}^{2} e\right\|_{1}\|\chi\|_{1} \\
& \leq C k^{2} h^{-1} \sup _{\left[r_{0}, R\right]}\left\|\partial_{r}^{2} e\right\|_{1}\|\chi\| \\
& \leq C k^{2} h^{m-1}\|\chi\|\left(\sum_{j=0}^{2} \sup _{\left[r_{0}, R\right]}\left\|\partial_{r}^{j} u\right\|_{m+1}\right), \quad \forall \chi \in S_{h}^{m} .
\end{aligned}
$$

Also, using (2.4), (2.2), (1.21), the Taylor formula and (2.5), we obtain

$$
\begin{aligned}
& \operatorname{Re}\left(T_{6,3}(\chi)\right)=\operatorname{Re} {\left[\mathrm{i} \frac{k^{2}}{8} \mathcal{B}\left(r^{n+\frac{1}{2}} ; \partial_{r}^{2} u\left(r^{n+\frac{1}{2}}\right), \chi\right)\right] } \\
&+\operatorname{Re}\left[\mathrm{i} \frac{k^{2}}{8} \mathcal{B}\left(r^{n+\frac{1}{2}} ; \mathcal{A} \sigma^{n}-\sigma\left(r^{n+\frac{1}{2}}\right), \chi\right)\right] \\
& \leq \operatorname{Re}\left[\mathrm{i} \frac{k^{2}}{8} \mathcal{B}\left(r^{n+\frac{1}{2}} ; \partial_{r}^{2} u\left(r^{n+\frac{1}{2}}\right), \chi\right)\right] \\
&+C k^{2}\|\chi\|_{1}\left\|\mathcal{A} \sigma^{n}-\sigma\left(r^{n+\frac{1}{2}}\right)\right\|_{1} \\
& \leq \operatorname{Re}\left[\mathrm{i} \frac{k^{2}}{8} \mathcal{B}\left(r^{n+\frac{1}{2}} ; \partial_{r}^{2} u\left(r^{n+\frac{1}{2}}\right), \chi\right)\right] \\
&+C k^{2} h^{-1}\|\chi\|\left\|\mathcal{A} \sigma^{n}-\sigma\left(r^{n+\frac{1}{2}}\right)\right\|_{1} \\
& \leq \operatorname{Re}\left[\mathrm{i} \frac{k^{2}}{8} \mathcal{B}\left(r^{n+\frac{1}{2}} ; \partial_{r}^{2} u\left(r^{n+\frac{1}{2}}\right), \chi\right)\right] \\
&+C k^{4} h^{-1}\|\chi\| \sup _{\left[r_{0}, R\right]}\left\|\partial_{r}^{2} \sigma\right\|_{1} \\
& \leq \operatorname{Re}\left[\mathrm{i} \frac{k^{2}}{8} \mathcal{B}\left(r^{n+\frac{1}{2}} ; \partial_{r}^{2} u\left(r^{n+\frac{1}{2}}\right), \chi\right)\right] \\
&+C k^{4} h^{-1}\|\chi\| \sum_{j=2}^{4} \sup _{\left[r_{0}, R\right]}\left\|\partial_{r}^{j} u\right\|_{1}
\end{aligned}
$$

for $\chi \in S_{h}^{m}$. Finally, observing that the first term in the bounds of $\operatorname{Re}\left(T_{6,3}(\chi)\right)$ and $\operatorname{Re}\left(T_{6,1}(\chi)\right)$ cancel out, we combine (3.13), (3.14), (3.15) and (3.16) to obtain (3.17)

$$
\operatorname{Re}\left(T_{6}(\chi)\right) \leq C\|\chi\|\left[k^{2} h^{m-1} \sum_{j=0}^{2} \sup _{\left[r_{0}, R\right]}\left\|\partial_{r}^{j} u\right\|_{m+1}+k^{4} h^{-1} \sum_{j=2}^{4} \sup _{\left[r_{0}, R\right]}\left\|\partial_{r}^{j} u\right\|_{1}\right]
$$


for $\chi \in S_{h}^{m}$. Thus, combining (3.8), (3.9), (3.10), (3.11), (3.12) and (3.17), we arrive at

$$
\sum_{j=1}^{6} \operatorname{Re}\left(T_{j}(\chi)\right) \leq C\|\chi\|\left[k^{2} B_{1}(u)+h^{m+1} B_{2}(u)+k^{4} h^{-1} B_{3}(u)\right]
$$

for $\chi \in S_{h}^{m}$, where

$$
\begin{aligned}
B_{1}(u) & :=\sup _{\left[r_{0}, R\right]}\left\|\partial_{r}^{3} u\right\|_{1}+\max _{0 \leq j \leq 2} \sup _{\left[r_{0}, R\right]}\left\|\partial_{r}^{j} u\right\|_{m+1}, \\
B_{2}(u) & :=\max _{0 \leq j \leq 1} \sup _{\left[r_{0}, R\right]}\left\|\partial_{r}^{j} u\right\|_{m+1}, \\
B_{3}(u) & :=\max _{2 \leq j \leq 4} \sup _{\left[r_{0}, R\right]}\left\|\partial_{r}^{j} u\right\|_{1} .
\end{aligned}
$$

From (3.7) and (3.18), we conclude that there exists a positive constant $C_{\Theta}$ such that

$$
\begin{aligned}
\left(1-C_{\Theta} k\right)\left\|\theta^{n+1}\right\| \leq(1 & \left.+C_{\Theta} k\right)\left\|\theta^{n}\right\| \\
& +C_{\Theta} k\left[k^{2} B_{1}(u)+h^{m+1} B_{2}(u)+k^{4} h^{-1} B_{3}(u)\right]
\end{aligned}
$$

for $n=0, \ldots, N-1$. Assuming that $C_{\Theta} k \leq \frac{1}{3}$ and applying a standard discrete Gronwall argument (cf. the proof of Proposition 3.4), from (3.20) we arrive at

$$
\max _{0 \leq n \leq N}\left\|\theta^{n}\right\| \leq C\left[\left\|\theta^{0}\right\|+k^{2} B_{1}(u)+h^{m+1} B_{2}(u)+k^{4} h^{-1} B_{3}(u)\right] .
$$

Now, we are ready to establish our error estimate in the theorem below.

Theorem 3.5. Let $\left(U^{n}\right)_{n=0}^{N}$ be the approximations derived by the numerical method (1.22) -(1.23). Also, we assume that $k^{2}=\mathcal{O}(h), C_{\Theta} k \leq \frac{1}{3}$ where $C_{\Theta}$ is the constant in (3.20), and there exists a constant $C_{\star}>0$ independent of $h$ such that

$$
\left\|u_{0, h}-u_{0}\right\| \leq C_{\star} h^{m+1}\left\|u_{0}\right\|_{m+1}, \quad \forall h \in\left(0, h_{\star}\right] .
$$

Then there exists a positive constant $C$ independent of $k$ and $h$ such that

$$
\max _{0 \leq n \leq N}\left\|U^{n}-u\left(r^{n}\right)\right\| \leq C\left[k^{2}\left(B_{1}(u)+B_{3}(u)\right)+h^{m+1} B_{2}(u)\right]
$$

where $B_{1}(u), B_{2}(u), B_{3}(u)$ are defined in (3.19).

Proof. First, we use (3.5), (3.21), (2.5) and (3.22) to conclude that

$$
\max _{0 \leq n \leq N}\left\|U^{n}-R_{h}\left(r^{n}\right) u\left(r^{n}\right)\right\| \leq C\left[k^{2} B_{1}(u)+h^{m+1} B_{2}(u)+k^{4} h^{-1} B_{3}(u)\right] .
$$

Then, we combine (3.24), (2.11) and the assumption $k^{2}=\mathcal{O}(h)$ to arrive at (3.23).

Remark 3.6. The error bound (3.22) is valid when $u_{0, h}$ is the $L^{2}(D)$ projection of $u_{0}$ onto $S_{h}^{m}$ or an $S_{h}^{m}$-interpolant of $u_{0}$. 


\section{NUMERICAL EXPERIMENTS}

In this section we report on the outcome of some numerical experiments performed with the fully discrete method of Section 1.7 .

In particular, we consider the ibvp (1.1)-(1.3) and (1.5), with the following data: $\left[\theta_{A}, \theta_{B}\right]=[0,22.5],\left[r_{0}, R\right]=[1,301], f_{0}=25 \mathrm{~Hz}, c(r, z, \theta)=c_{0}=1500 \mathrm{~m} / \mathrm{s}$ so that $n_{R F}(r, z, \theta)=1$, a variable bottom topography represented by the function

$$
s(r, \theta)=100+\sin \left(\frac{\pi r}{60}\right) \exp \left(-4 k_{0}\left(\theta-\theta_{M}\right)^{2}\right) \quad \forall(r, \theta) \in\left[r_{0}, R\right] \times\left[\theta_{A}, \theta_{B}\right],
$$

where $\theta_{M}:=\frac{\theta_{A}+\theta_{B}}{2}$, and an initial condition given by

$$
\psi_{0}(z, \theta)=\sqrt{\frac{k_{0}}{2}} \phi_{0}\left(z ; z_{S}\right) \phi_{0}\left(\theta ; \theta_{M}\right),
$$

where

$$
\phi_{0}(x ; p):=\exp \left(-\frac{k_{0}^{2}}{4}(x-p)^{2}\right)-\exp \left(-\frac{k_{0}^{2}}{4}(x+p)^{2}\right)
$$

and $z_{S}=50 \mathrm{~m}$ is the depth where the point harmonic source is placed. The latter function is known as an analytical starter or generalized Gaussian source and is widely used in underwater acoustic simulations. It can be shown that (4.2) is a far field approximation of a point-source solution of the Helmholtz equation in a homogeneous medium; see, e.g. [19].

Our working example will be the ibvp (1.7) which follows after performing the transformation (1.6) and then adding a forcing term $f$ which follows after choosing an exact solution $u$ that satisfies the initial and the boundary conditions.

We implemented the Crank-Nicolson finite element scheme (1.22)-(1.23) using uniform, rectangular partitions of the domain $\overline{\mathcal{D}}$ of maximum diameter $h$. The family $S_{h}^{m}$ of finite-dimensional subspaces of $C(\overline{\mathcal{D}}) \cap \mathbb{H}^{1}(\mathcal{D})$ was that of piecewise linear functions on each element of the partition, i.e. $m=1$. The starting approximation $U^{0}$ was chosen as the interpolant in $S_{h}^{1}$ of the initial condition (4.1).

Our first set of experiments concerns the experimental verification of the convergence rate of the scheme in the spatial variables. The measure of the error was $E(r):=\left\|u(r)-U^{n}\right\|$ for $r=n k$ and $n \in \mathbb{N}$, whereas for other values of $r E(r)$ was defined by linear interpolation. To determine experimentally the spatial order of convergence, the approximate solution was computed for $1 \leq r \leq 301$ using a rectangular partition of $\overline{\mathcal{D}}$ with the inverse element diameter ranging from 96 to 384 . For these runs, very small $r$-steps were taken to ensure that the error due to the discretization in range variable $r$ is negligible. The observed error was recorded at $r=101,201$ and 301. As usual, the convergence rate corresponding to two different runs with mesh sizes $h_{1}, h_{2}$ and corresponding errors $E_{1}$ and $E_{2}$ is defined to be $\log \left(\frac{E_{1}}{E_{2}}\right) / \log \left(\frac{h_{1}}{h_{2}}\right)$. The results are shown in Table 1. It is evident that the convergence rate of the spatial component of the error is indeed two.

The determination of the accuracy in the range variable $r$ is more delicate. We took $h^{-1}=192$ and computed the solution of our problem up to $r=301$ for various values of $k$. For this fixed value of $h$ we made a reference calculation with a small value of $k=k_{\text {ref }}=\frac{h}{20}$. The corresponding approximate solution, denoted by $U_{h, \text { ref }}$, differs from the exact solution by a factor which is almost entirely due to the spatial discretization. We then define a modified measure of the error $E^{*}(r)$ as above but with the exact solution replaced by the reference solution $U_{h, \text { ref }}$. The results are shown in Table 2 . 
TABLE 1. Errors $E(r)$ and spatial convergence rate for $k^{-1}=768$

\begin{tabular}{r|cc|cc|cc}
\hline & \multicolumn{2}{|c|}{$r=101$} & \multicolumn{2}{c|}{$r=201$} & \multicolumn{2}{c}{$r=301$} \\
\hline$h^{-1}$ & $E(r)$ & Rate & $E(r)$ & Rate & $E(r)$ & Rate \\
\hline 96 & $1.3227(-1)$ & & $1.5485(-1)$ & & $1.9143(-1)$ & \\
\hline 192 & $2.6685(-2)$ & 2.31 & $3.1024(-2)$ & 2.32 & $3.8105(-2)$ & 2.32 \\
\hline 256 & $1.4016(-2)$ & 2.24 & $1.6232(-2)$ & 2.25 & $1.9873(-2)$ & 2.26 \\
\hline 288 & $1.0895(-2)$ & 2.15 & $1.2575(-2)$ & 2.17 & $1.5312(-2)$ & 2.21 \\
\hline 384 & $6.0073(-3)$ & 2.07 & $6.8971(-3)$ & 2.09 & $8.2997(-3)$ & 2.13 \\
\hline
\end{tabular}

TABLE 2. Errors $E(r)$ and $r$-convergence rate for $h^{-1}=192$ and $r=301$

\begin{tabular}{c|ccc}
\hline$k^{-1}$ & $E(r)$ & $E^{*}(r)$ & Rate \\
\hline 192 & $9.9887(-1)$ & $0.1304(+1)$ & \\
\hline 384 & $1.7834(-1)$ & $2.2936(-1)$ & 2.51 \\
\hline 512 & $9.2327(-2)$ & $1.2048(-1)$ & 2.24 \\
\hline 576 & $7.1442(-2)$ & $9.3765(-2)$ & 2.13 \\
\hline
\end{tabular}

\section{A global Elliptic REgUlarity RESUlt}

In this section, we present a general Global Elliptic Regularity Theorem for complex elliptic operators with mixed Dirichlet-Robin boundary conditions, in rectangles of $\mathbb{R}^{2}$. Our proof follows that of [15, which deals with the Dirichlet problem for real operators. In our approach, the main idea is that if the trace terms in the weak formulation of the problem vanish due to the boundary conditions, for suitably chosen test functions, then a Global Elliptic Regularity result is proved in Theorem 5.1. Note that the Robin condition in this theorem does not involve any zero order term, while the first order terms are related to the coefficients of the boundary problem so that indeed in the weak formulation, after integration by parts, the trace integrals vanish. Our result is established by the fact that the closure of a rectangle can be covered by using a finite union of half-balls together with an open smooth domain in the interior. We then apply an exponential transformation and extent our result, in Theorem 5.3. where an arbitrary zero order term is introduced at the Robin condition of Theorem 5.1

Theorem 5.1. Let $\mathcal{W}=(0,1) \times\left(\theta_{A}, \theta_{B}\right)$ be a rectangular domain in cartesian coordinates. We consider the following boundary value problem: We seek a complexvalued function $u$ such that

$$
\begin{aligned}
& A u_{z z}+B u_{z \theta}+C u_{\theta \theta}+D u_{z}+E u_{\theta}+F u=f \quad \text { in } \quad \mathcal{W} \\
& u(0, \theta)=0 \\
& u\left(z, \theta_{A}\right)=u\left(z, \theta_{B}\right)=0, \\
& a(\theta) u_{z}+b(\theta) u_{\theta}=0 \quad \text { at } \quad z=1,
\end{aligned}
$$

where $A, B, C \in C^{1}(\overline{\mathcal{W}}), D, E, F \in L^{\infty}(\mathcal{W}), f \in L^{2}(\mathcal{W}), a:\left[\theta_{A}, \theta_{B}\right] \rightarrow \mathbb{C} \backslash\{0\}$ and $b:\left[\theta_{A}, \theta_{B}\right] \rightarrow \mathbb{C}$. We also assume that $A, B, C$ take imaginary values and $\frac{A}{\mathrm{i}}, \frac{C}{\mathrm{i}}$ are 


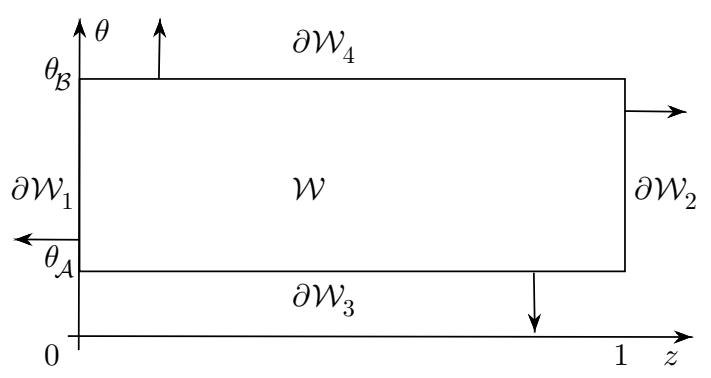

FIGURE 2. The rectangular domain $\mathcal{W}$.

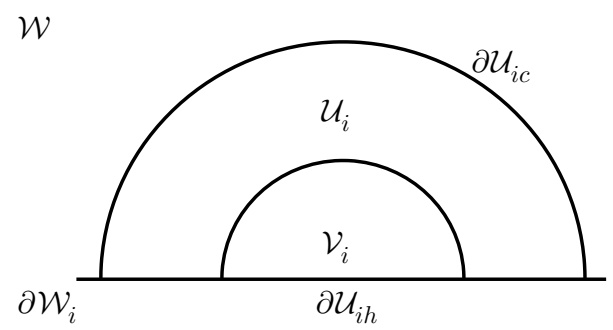

FiguRE 3. Half-balls, curved boundary, horizontal boundary.

always positive (or always negative). Moreover, we assume that

$$
\begin{gathered}
|A C|>\frac{|B|^{2}}{4}, \quad \text { for any }(z, \theta) \in \mathcal{W}, \\
2 A(1, \theta) b(\theta)=B(1, \theta) a(\theta), \quad \text { for any } \theta \in\left[\theta_{A}, \theta_{B}\right] .
\end{gathered}
$$

If $u \in H^{1}(\mathcal{W})$ is a weak solution of (5.1), then the following elliptic regularity estimate holds:

$$
u \in H^{2}(\mathcal{W}) \quad \text { and } \quad\|u\|_{H^{2}(\mathcal{W})} \leq c\|f\|_{L^{2}(\mathcal{W})} .
$$

Proof. We consider the rectangle $\mathcal{W}$. Obviously its boundary is the union of four linear segments and we write $\partial \mathcal{W}=\bigcup_{i=1}^{4} \partial \mathcal{W}_{i}$ (cf. Figure 2). Let $\mathcal{U}_{i}=B^{o}\left(k_{i}, r_{i}\right) \cap$ $\overline{\mathcal{W}}$ be a half-ball in $\mathbb{R}^{2}$ in $\overline{\mathcal{W}}$ laying at $\partial \mathcal{W}$ of range $r_{i}$ and of diameter in $\partial \mathcal{W}$. We define its boundary by $\partial \mathcal{U}_{i}:=\partial \mathcal{U}_{i h} \cup \partial \mathcal{U}_{i c}$, where $\partial \mathcal{U}_{i h}$ is the diameter such that $\partial \mathcal{U}_{i h} \subseteq \partial \mathcal{W}_{i}$, and $\partial \mathcal{U}_{i c}$ is the semicircle of range $r_{i}$ such that $\mathcal{U}_{i} \subset \overline{\mathcal{W}}$. We also consider $\mathcal{V}_{i}=B^{o}\left(k_{i}, r_{i} / 2\right) \cap \overline{\mathcal{W}}$, the half-ball being of the same center $k_{i}$ as $\mathcal{U}_{i}$ and of range $r_{i} / 2$ (cf. Figure 3 ). Obviously, $\partial \mathcal{W}$ is compact, thus $\partial \mathcal{W}$ may be covered by using a finite union of sets of the form $\mathcal{V}_{i}$, while the same union together with a suitably chosen smooth domain in $\mathcal{W}$ covers $\overline{\mathcal{W}}$. By [15] an interior regularity estimate holds. Our aim is to prove the regularity estimate

$$
\|u\|_{H^{2}\left(\mathcal{V}_{i}\right)} \leq c\|f\|_{L^{2}\left(\mathcal{U}_{i}\right)}, \quad i=1, \ldots, 4 .
$$

Interior regularity combined with the estimate (5.5) gives the desired result (5.4) (cf. [15], pg. 322). 
We consider $\phi_{i} \in H^{1}\left(\mathcal{U}_{i}\right)$ and let $u$ be the weak solution of (5.1). If $(u, v)_{\mathcal{U}_{i}}:=$ $\int_{\mathcal{U}_{i}} u \bar{v} d s$, then using the differential equation of problem (5.1) and integrating by parts we have

$$
\begin{aligned}
\left(f, \phi_{i}\right)_{\mathcal{U}_{i}}= & -\left(A u_{z}, \partial_{z} \phi_{i}\right)_{\mathcal{U}_{i}}-\left\{\left(\frac{B}{2} u_{z}, \partial_{\theta} \phi_{i}\right)_{\mathcal{U}_{i}}+\left(\frac{B}{2} u_{\theta}, \partial_{z} \phi_{i}\right)_{\mathcal{U}_{i}}\right\} \\
& -\left(C u_{\theta}, \partial_{\theta} \phi_{i}\right)_{\mathcal{U}_{i}}+\left(\widetilde{D} u_{z}, \phi_{i}\right)_{\mathcal{U}_{i}}+\left(\widetilde{E} u_{\theta}, \phi_{i}\right)_{\mathcal{U}_{i}}+\left(F u, \phi_{i}\right)_{\mathcal{U}_{i}} \\
& +\int_{\partial \mathcal{U}_{i}}\left[u_{z}\left(A, \frac{B}{2}\right)+u_{\theta}\left(\frac{B}{2}, C\right)\right] \bar{\phi}_{i} \overrightarrow{\eta_{i}} d s
\end{aligned}
$$

where $\widetilde{D}=-A_{z}-\frac{B_{\theta}}{2}+D, \widetilde{E}=-C_{\theta}-\frac{B_{z}}{2}+E$ are the resulting terms after integration by parts, and $\vec{\eta}_{i}$ is the outward unit normal to $\partial \mathcal{U}_{i}$. We let $\Omega_{i}\left(u, \phi_{i}\right):=$ $\int_{\partial \mathcal{U}_{i}}\left[u_{z}\left(A, \frac{B}{2}\right)+u_{\theta}\left(\frac{B}{2}, C\right)\right] \bar{\phi}_{i} \vec{\eta}_{i} d s$, and define the vector $K_{i}:=\left[u_{z}\left(A, \frac{B}{2}\right)+u_{\theta}\left(\frac{B}{2}, C\right)\right] \bar{\phi}_{i} ;$ here $(\cdot, \cdot)$ denotes a vector of $\mathbb{R}^{2}$. Then for $\partial \mathcal{U}_{i}=\partial \mathcal{U}_{i h} \cup \partial \mathcal{U}_{i c}$ it holds that $\Omega_{i}\left(u, \phi_{i}\right)=$ $\int_{\partial \mathcal{U}_{i h}} K_{i} \vec{\eta}_{i} d s+\int_{\partial \mathcal{U}_{i c}} K_{i} \vec{\eta}_{i} d s$. Using the boundary conditions of $u \in H^{1}(\mathcal{W})$ we obtain

$$
\begin{aligned}
& \Omega_{1}\left(u, \phi_{1}\right)=-\int_{\partial \mathcal{U}_{1 h}} A u_{z} \bar{\phi}_{1} d s+\int_{\partial \mathcal{U}_{1 c}} K_{1} \overrightarrow{\eta_{1}} d s \\
& \Omega_{2}\left(u, \phi_{2}\right)=\int_{\partial \mathcal{U}_{2 c}} K_{2} \overrightarrow{\eta_{2}} d s \\
& \Omega_{3}\left(u, \phi_{3}\right)=-\int_{\partial \mathcal{U}_{3 h}} C u_{\theta} \bar{\phi}_{3} d s+\int_{\partial \mathcal{U}_{3 c}} K_{3} \overrightarrow{\eta_{3}} d s \\
& \Omega_{4}\left(u, \phi_{4}\right)=\int_{\partial \mathcal{U}_{4 h}} C u_{\theta} \bar{\phi}_{4} d s+\int_{\partial \mathcal{U}_{4 c}} K_{4} \overrightarrow{\eta_{4}} d s .
\end{aligned}
$$

We note that for the term $\Omega_{2}\left(u, \phi_{2}\right)$ the boundary condition along $z=1$ is crucial. More specifically,

$$
\Omega_{2}\left(u, \phi_{2}\right)=\int_{\partial \mathcal{U}_{2 h}} K_{2} \overrightarrow{\eta_{2}} d s+\int_{\partial \mathcal{U}_{2 c}} K_{2} \overrightarrow{\eta_{2}} d s
$$

But we observe that $\partial \mathcal{U}_{2 h} \subset \partial \mathcal{W}_{2}$ (where $z=1$ ). So, using the definition of $K_{2}$, i.e.

$$
K_{2}=\left[u_{z}\left(A, \frac{B}{2}\right)+u_{\theta}\left(\frac{B}{2}, C\right)\right] \bar{\phi}_{2}=\bar{\phi}_{2}\left(u_{z} A+u_{\theta} \frac{B}{2}, u_{z} \frac{B}{2}+u_{\theta} C\right),
$$

and the boundary condition along $z=1$ which gives $u_{z} A+u_{\theta} \frac{B}{2}=0$ on $\partial \mathcal{U}_{2 h}$, we obtain

$$
\int_{\partial \mathcal{U}_{2 h}} K_{2} \overrightarrow{\eta_{2}} d s=\int_{\partial \mathcal{U}_{2 h}} \overline{\phi_{2}}\left(0, u_{z} \frac{B}{2}+u_{\theta} C\right) \overrightarrow{\eta_{2}} d s=0
$$

since the second coordinate of $\overrightarrow{\eta_{2}}$ is zero (cf. Figure 2). Thus indeed we have

$$
\Omega_{2}\left(u, \phi_{2}\right)=\int_{\partial \mathcal{U}_{2 c}} K_{2} \overrightarrow{\eta_{2}} d s .
$$

Our aim now is to find test functions $\phi_{i}$ such that in the weak formulation the trace terms vanish.

Assumption 1. We assume that there exist functions $\phi_{i}$ that satisfy the following requirements:

- The test functions are smooth and along the curved boundary $\mathcal{U}_{i c}$ of $\mathcal{U}_{i}$ vanish: $\phi_{i} \in H^{1}\left(\mathcal{U}_{i}\right)$, and $\phi_{i}=0$ on $\partial \mathcal{U}_{i c}, i=1, \ldots, 4$. 
- For $i=1,3,4$, the test functions also vanish along the horizontal boundary $\mathcal{U}_{i h}$ of $\mathcal{U}_{i}: \phi_{1}=0$ at $z=0, \phi_{2}$ is arbitrary, $\phi_{3}=0$ at $\theta=\theta_{A}, \phi_{4}=0$ at $\theta=\theta_{B}$.

Under this assumption, the sum of trace integrals in the weak formulation equals zero because $\Omega_{i}\left(u, \phi_{i}\right)=0$ for any $i=1, \ldots, 4$. The weak formulation (5.6) for $\mathcal{B}\left(u, \phi_{i}\right)_{\mathcal{U}_{i}}:=\left(f, \phi_{i}\right)_{\mathcal{U}_{i}}$ becomes

$$
\begin{aligned}
\mathcal{B}\left(u, \phi_{i}\right)_{\mathcal{U}_{i}}= & -\left(A u_{z}, \partial_{z} \phi_{i}\right)_{\mathcal{U}_{i}}-\left\{\left(\frac{B}{2} u_{z}, \partial_{\theta} \phi_{i}\right)_{\mathcal{U}_{i}}+\left(\frac{B}{2} u_{\theta}, \partial_{z} \phi_{i}\right)_{\mathcal{U}_{i}}\right\} \\
& -\left(C u_{\theta}, \partial_{\theta} \phi_{i}\right)_{\mathcal{U}_{i}}+\left(\widetilde{D} u_{z}, \phi_{i}\right)_{\mathcal{U}_{i}}+\left(\widetilde{E} u_{\theta}, \phi_{i}\right)_{\mathcal{U}_{i}}+\left(F u, \phi_{i}\right)_{\mathcal{U}_{i}} .
\end{aligned}
$$

The next step is to define, properly, for any $i=1, \cdots, 4$, test functions $\phi_{i}$ satisfying this assumption. We define the following general cut-off function ([15]):

$$
J=\left\{\begin{array}{l}
0 \text { in } \mathbb{R}^{2}-B(\tilde{l}, r), \\
1 \text { in } B(\tilde{l}, r / 2), \\
\left.0 \leq J \leq 1 \text { elsewhere (with } J=0 \text { near } \partial \mathcal{U}_{c}\right) .
\end{array}\right.
$$

Here $\mathcal{U}:=B^{o}(\tilde{l}, r) \cap \overline{\mathcal{W}}$ is a half-ball in $\mathbb{R}^{2}$ of radius $r$ and of center $\tilde{l}$ such that $\partial \mathcal{U}_{h} \subseteq \partial \mathcal{W}$. Let $\mathcal{V}$ be the half-ball in $\mathbb{R}^{2}$ of center $\tilde{l}$ and of range $r / 2$ with diameter in $\partial \mathcal{U}_{h}$. Obviously the cut-off function $J$ in $\mathcal{V}$ equals 1 , and near $\partial \mathcal{U}_{c}$ is 0 . Let $\tilde{u}$ be a function in $H^{1}(\mathcal{W})$ that satisfies the boundary conditions of problem (5.1). We define the function $([15])$

$$
\tilde{v}:=-D^{-h}\left(J^{2} D^{h} \tilde{u}\right) \text {, with } D^{h} \tilde{u}(x):=\frac{\tilde{u}(x+h e)-\tilde{u}(x)}{h}, x \in \mathcal{U},
$$

where $h$ is a positive number and $e$ is a unitary vector (direction) in $\mathbb{R}^{2}$ parallel to the diameter of the half-ball $\mathcal{U}$.

In this way for every boundary line $(i=1, \cdots, 4)$ of the rectangular domain $\mathcal{W}$ we define a cut-off function $J_{i}$ and denote by $e_{i}$ the unitary direction of the specific boundary line $\partial \mathcal{W}_{i}$. We then prove first that $\tilde{v}_{i}$ defined by these $J_{i}$ in (5.10) for the directions $e_{i}$ are test functions that satisfy Assumption 1, and in the sequel we set $\phi_{i}:=\tilde{v}_{i}$.

More specifically, for every $i=1, \ldots, 4$ we consider $\mathcal{U}_{i}=B^{o}\left(k_{i}, r_{i}\right) \cap \overline{\mathcal{W}}, \quad \mathcal{V}_{i}=$ $B^{0}\left(k_{i}, \frac{r_{i}}{2}\right) \cap \overline{\mathcal{W}}, \quad k_{i}, r_{i}$ such that $\mathcal{U}_{i} \subseteq \mathcal{W}, \partial \mathcal{U}_{i h} \subseteq \partial \mathcal{W}_{i}$ and define the cut-off function

$$
J_{i}:=\left\{\begin{array}{l}
J_{i}=0 \text { in } \mathbb{R}^{2}-B\left(k_{i}, r_{i}\right), \\
J_{i}=1 \text { in } B\left(k_{i}, \frac{r_{i}}{2}\right), \\
\left.0 \leq J_{i} \leq 1 \text { elsewhere (with } J_{i}=0 \text { near } \partial \mathcal{U}_{i c}\right)
\end{array}\right.
$$

Let $\tilde{u}$ be a function in $H^{1}(\mathcal{W})$ that satisfies the boundary conditions of problem (5.1); we define as previously the function

$$
\tilde{v}_{i}:=-D_{i}^{-h}\left(J_{i}^{2} D_{i}^{h} \tilde{u}\right), \text { with } D_{i}^{h} \tilde{u}(x):=\frac{\tilde{u}\left(x+h e_{i}\right)-\tilde{u}(x)}{h}, x \in \mathcal{U}_{i} .
$$

By [15], for any $x \in \mathcal{U}_{i}$, the following identity holds:

$$
\tilde{v}_{i}(x)=-\frac{1}{h^{2}}\left(J_{i}^{2}\left(x-h e_{i}\right)\left[\tilde{u}(x)-\tilde{u}\left(x-h e_{i}\right)\right]-J_{i}^{2}(x)\left[\tilde{u}\left(x+h e_{i}\right)-\tilde{u}(x)\right]\right) .
$$

Using the boundary conditions of the elliptic problem and the identity (5.12), we will prove that $\tilde{v}_{i}$ satisfy Assumption 1 for any $i=1, \ldots, 4$.

If $i=1$, then obviously $\tilde{v}_{1}$ is in $H^{1}\left(\mathcal{U}_{1}\right)$. We notice that if $x$ is in $\partial \mathcal{U}_{1 c}$ then $J_{1}(x)=0$ and for $h$ small enough $J_{1}\left(x-h e_{1}\right)=0$, so by (5.12) $\tilde{v}_{1}(x)=0$ on $\partial \mathcal{U}_{1 c}$. 
Along the boundary line $\partial \mathcal{U}_{1 h}$ it holds that $z=0$ and $e_{1}=(0,1)$. If $x=(0, \theta)$, then $\tilde{u}(x)=0$ and $\tilde{u}\left(x \pm h e_{1}\right)=\tilde{u}(0, \theta \pm h)=0$; thus by (5.12) it follows that $\tilde{v}_{1}(0, \theta)=0$.

If $i=2$, then $\tilde{v}_{2}(x) \in H^{1}\left(\mathcal{U}_{2}\right)$, and $e_{2}=(0,1)$. If $x \in \partial \mathcal{U}_{2 c}$, then for $h$ small $J_{2}(x)=J_{2}\left(x-h e_{2}\right)=0$; thus by (5.12) $\tilde{v}_{2}(x)=0$ on $\partial \mathcal{U}_{2 c}$.

If $i=3$, then $\tilde{v}_{3}(x) \in H^{1}\left(\mathcal{U}_{3}\right)$ and $e_{3}=(1,0)$, for $h$ small. If $x \in \mathcal{U}_{3 c}$, then $J_{3}(x)=J_{3}\left(x-h e_{3}\right)=0$; thus $\tilde{v}_{3}(x)=0$ on $\partial \mathcal{U}_{3 c}$. For $x=\left(z, \theta_{A}\right)$ then $\tilde{u}(x)=$ $\tilde{u}\left(z, \theta_{A}\right)=0$ and $\tilde{u}\left(x \pm h e_{3}\right)=\tilde{u}\left(z \pm h, \theta_{A}\right)=0$. By (5.12) it follows that $\tilde{v}_{3}\left(z, \theta_{A}\right)=0$.

If $i=4$, then $\tilde{v}_{4}(x) \in H^{1}\left(\mathcal{U}_{4}\right)$ and $e_{4}=(1,0)$. If $x$ is in $\partial \mathcal{U}_{4 c}$, then $J_{4}(x)=0$ and for $h$ small enough $J_{4}\left(x-h e_{4}\right)=0$; thus by (5.12) $\tilde{v}_{4}(x)=0$ on $\partial \mathcal{U}_{4 c}$. If $x=\left(z, \theta_{B}\right)$, then $\tilde{u}(x)=\tilde{u}\left(z, \theta_{B}\right)=0$ and $\tilde{u}\left(x \pm h e_{4}\right)=\tilde{u}\left(z \pm h, \theta_{B}\right)=0$; thus $\tilde{v}_{4}\left(z, \theta_{B}\right)=0$.

Therefore, in all cases Assumption 1 holds and the trace terms vanish from the weak formulation of the elliptic problem. If we set $\tilde{u}:=u$, where $u$ is the weak solution of the elliptic problem satisfying the boundary conditions, then it can be easily proved (for details see [6] and [15]) by use of ellipticity, the weak formulation and the boundary conditions at $z=0, \theta=\theta_{A}, \theta=\theta_{B}$, that for every half-ball $\mathcal{V}_{i}$ it holds that

$$
\|u\|_{H^{2}\left(\mathcal{V}_{i}\right)} \leq c\left[\|f\|_{L^{2}\left(\mathcal{U}_{i}\right)}+\|u\|_{H^{1}\left(\mathcal{U}_{i}\right)}\right] .
$$

The finite summation of (5.13) ) over any $\mathcal{V}_{i}$ (of type $i=1, \ldots, 4$ ) and the interior regularity give $([15])$

$$
\|u\|_{H^{2}(\mathcal{W})} \leq c\left[\|f\|_{L^{2}(\mathcal{W})}+\|u\|_{H^{1}(\mathcal{W})}\right] .
$$

Combining (5.14) with ellipticity we obtain the elliptic regularity result

$$
\|u\|_{H^{2}(\mathcal{W})} \leq c\|f\|_{L^{2}(\mathcal{W})} .
$$

Remark 5.2. We note that an analogous result is also valid if in the assumptions of Theorem 5.1 the homogeneous condition at $z=1$ is replaced by the nonhomogeneous condition $a(\theta) u_{z}+b(\theta) u_{\theta}=g$ at $z=1$, for any $g \in H^{\frac{1}{2}}\left(\partial \mathcal{W}_{R}\right)$, where $\partial \mathcal{W}_{R}=\{1\} \times\left(\theta_{A}, \theta_{B}\right)$. In this case, in the weak formulation the trace integral term containing $g$ is hidden due to ellipticity, leaving at the right-hand side of (5.15) the extra term $c|g|_{\frac{1}{2}, \partial \mathcal{W}_{R}}$ where

$$
|g|_{\frac{1}{2}, \partial \mathcal{W}_{R}}:=\inf \left\{\|v\|_{H^{1}(\mathcal{W})}: v \in H^{1}(\mathcal{W}),\left.v\right|_{\partial \mathcal{W}_{R}}=g \text { and } v_{\partial \mathcal{W} \backslash \partial \mathcal{W}_{R}}=0\right\} .
$$

More specifically, the following elliptic regularity estimate holds:

$$
\|u\|_{H^{2}(\mathcal{W})} \leq c\left(\|f\|_{L^{2}(\mathcal{W})}+|g|_{\frac{1}{2}, \partial \mathcal{W}_{R}}\right) .
$$

The following theorem extends Theorem 5.1 in the sense that we can add at the boundary condition along $z=1$ a zero order term multiplied by an arbitrary smooth function $c(\theta)$.

Theorem 5.3. Under the assumptions of Theorem 5.1, if the boundary condition of (5.1) at $z=1$ has the form

$$
a(\theta) u_{z}+b(\theta) u_{\theta}+c(\theta) u(\theta)=0 \text { at } z=1, \theta \in\left[\theta_{A}, \theta_{B}\right],
$$

with $c$ a smooth complex function of $\theta$, then the results of Theorem 5.1 hold (elliptic regularity). 
Proof. We set $q=q(z, \theta)$ and consider the elliptic operator of (5.1). We apply the transformation $u:=\exp (q) w$ and get the following equivalent problem:

$$
\begin{aligned}
& A w_{z z}+B w_{z \theta}+C w_{\theta \theta}+D_{w} w_{z}+E_{w} w_{\theta}+F_{w} w=f_{w} \text { in } \mathcal{W}, \\
& w(0, \theta)=0, \\
& w\left(z, \theta_{A}\right)=w\left(z, \theta_{B}\right)=0, \\
& a(\theta) w_{z}+b(\theta) w_{\theta}+c_{w}(\theta) w(\theta)=0 \quad \text { at } z=1,
\end{aligned}
$$

where $D_{w}=2 A q_{z}+B q_{\theta}+D, E_{w}=B q_{z}+2 C q_{\theta}+E, f_{w}=\exp (-q) f, F_{w}=$ $F+A\left(q_{z z}+q_{z}^{2}\right)+B\left(q_{z \theta}+q_{z} q_{\theta}\right)+C\left(q_{\theta \theta}+q_{\theta}^{2}\right)+D q_{z}+E q_{\theta}$, and $c_{w}(\theta)=a(\theta) q_{z}+$ $b(\theta) q_{\theta}+c(\theta)$. We chose $q(z, \theta)$ such that $c_{w}(\theta)=0$ or equivalently

$$
a(\theta) q_{z}(1, \theta)+b(\theta) q_{\theta}(1, \theta)+c(\theta)=0 \text { for any } \theta \in\left[\theta_{A}, \theta_{B}\right] .
$$

The relation (5.19) can be achieved since $\frac{b}{a}$ is real and smooth, and $a, b$ cannot be simultaneously zero, [16]. Thus by (5.18) and (5.19) the problem is of the form covered by Theorem [5.1] and consequently

$$
w \in H^{2}(\mathcal{W}) \quad \text { and } \quad\|w\|_{H^{2}(\mathcal{W})} \leq c\left\|f_{w}\right\|_{L^{2}(\mathcal{W})} .
$$

Obviously $u=\exp (q) w$; therefore, $u \in H^{2}(\mathcal{W})$ and $\|u\|_{H^{2}(\mathcal{W})} \leq c\|f\|_{L^{2}(\mathcal{W})}$.

Remark 5.4. By using Remark [5.2, under the assumptions of Theorem 5.3] and if we impose the non-homogeneous condition $a(\theta) u_{z}+b(\theta) u_{\theta}+c(\theta) u(\theta)=g$ at $z=1$, for $g \in H^{\frac{1}{2}}\left(\partial \mathcal{W}_{R}\right)$ in place of the homogeneous one, estimate (5.16) follows (the proof is the same as in Theorem [5.3).

Remark 5.5. Theorem 5.1 and 5.3 or the results of Remarks 5.2 5.4 can be applied to cylindrical coordinates for $r$ fixed when $\mathcal{W}=\left\{(z, r, \theta) \in \mathbb{R}^{3}\right\}$, by use of the change of variables $u(z, \theta)=\hat{u}(z, \hat{\theta})$ with $\hat{\theta}:=\frac{2 \pi r \theta}{360}=c_{0} \theta$; then the equivalent problem in cartesian coordinates is defined in a rectangular domain and satisfies the assumptions of Theorems 5.1 and 5.3 or those of Remarks 5.2 and 5.4

\section{ACKNOWLEDGMENTS}

D. C. Antonopoulou acknowledges the support of the National Scholarship Foundation of Greece (Postdoctoral Research in Greece) and her advisor Professor V. A. Dougalis for proposing this problem that was partially analyzed in her Ph.D. Thesis. D. C. Antonopoulou and G. D. Karali are supported under the "ARISTEIA" Action of the "Operational programme education and lifelong learning" and is cofunded by the European Social Fund (ESF) and National Resources. M. Plexousakis and G. E. Zouraris have been partially supported by the European Union Seventh Framework Programme (FP7-REGPOT-2009-1) under the Grant Agreement no. 245749 (Archimedes Center for Modeling, Analysis and Computation, University of Crete, Greece).

\section{REFERENCES}

[1] Leif Abrahamsson and Heinz-Otto Kreiss, The initial-boundary value problem for the Schrödinger equation, Math. Methods Appl. Sci. 13 (1990), no. 5, 385-390, DOI 10.1002/mma.1670130503. MR.1078588 (91h:35093)

[2] Leif Abrahamsson and Heinz-Otto Kreiss, Boundary conditions for the parabolic equation in a range-dependent duct, J. Acoust. Soc. Amer. 87 (1990), no. 6, 2438-2441, DOI 10.1121/1.399089. MR 1054995 (91b:76090) 
[3] Georgios D. Akrivis and Vassilios A. Dougalis, Finite difference discretization with variable mesh of the Schrödinger equation in a variable domain, Bull. Soc. Math. Grèce (N.S.) 31 (1990), 19-28. MR 1108904 (93a:65111)

[4] G. D. Akrivis, V. A. Dougalis, and G. E. Zouraris, Error estimates for finite difference methods for a wide-angle "parabolic" equation, SIAM J. Numer. Anal. 33 (1996), no. 6, 2488-2509, DOI 10.1137/S0036142994266352. MR1427476 (98a:65110)

[5] G. D. Akrivis, V. A. Dougalis, and G. E. Zouraris, Finite difference schemes for the "parabolic" equation in a variable depth environment with a rigid bottom boundary condition, SIAM J. Numer. Anal. 39 (2001), no. 2, 539-565 (electronic), DOI 10.1137/S0036142999367460. MR1860264 (2002g:65090)

[6] D. C. Antonopoulou, Theory and numerical analysis of parabolic approximations, Ph.D. Thesis, University of Athens, Greece, 2006 (in Greek).

[7] D. C. Antonopoulou, V. A. Dougalis, F. Sturm and G. E. Zouraris, Conservative initialboundary value problems for the wide-angle PE in waveguides with variable bottoms, Proceedings of the 9th European Conference on Underwater Acoustics (9th EQUA), M. E. Zakharia, D. Cassereau and F. Luppé, eds. 1, 375-380 (2008).

[8] D. C. Antonopoulou, V. A. Dougalis, and G. E. Zouraris, Galerkin methods for parabolic and Schrödinger equations with dynamical boundary conditions and applications to underwater acoustics, SIAM J. Numer. Anal. 47 (2009), no. 4, 2752-2781, DOI 10.1137/070710858. MR:2551145(2010m:65212)

[9] Dimitra C. Antonopoulou, Vassilios A. Dougalis, and Georgios E. Zouraris, A finite difference method for the wide-angle "parabolic" equation in a waveguide with downsloping bottom, Numer. Methods Partial Differential Equations 29 (2013), no. 4, 1416-1440, DOI 10.1002/num.21762. MR 3053872

[10] D. C. Antonopoulou and M. Plexousakis, Discontinuous Galerkin methods for the linear Schrödinger equation in non-cylindrical domains, Numer. Math. 115 (2010), no. 4, 585-608, DOI 10.1007/s00211-010-0296-5. MR2658156(2011e:65184)

[11] A. Bamberger, B. Engquist, L. Halpern, and P. Joly, Parabolic wave equation approximations in heterogenous media, SIAM J. Appl. Math. 48 (1988), no. 1, 99-128, DOI 10.1137/0148005. MR.923293(89h:35225)

[12] Susanne C. Brenner and L. Ridgway Scott, The Mathematical Theory of Finite Element Methods, 3rd ed., Texts in Applied Mathematics, vol. 15, Springer, New York, 2008. MR2373954(2008m:65001)

[13] V. A. Dougalis, F. Sturm, and G. E. Zouraris, On an initial-boundary value problem for a wide-angle parabolic equation in a waveguide with a variable bottom, Math. Methods Appl. Sci. 32 (2009), no. 12, 1519-1540, DOI 10.1002/mma.1097. MR2535860 (2011a:35212)

[14] Todd Dupont, $L^{2}$-estimates for Galerkin methods for second order hyperbolic equations, SIAM J. Numer. Anal. 10 (1973), 880-889. MR0349045 (50 \#1539)

[15] L. C. Evans, Partial Differential Equations, Graduate Studies in Mathematics, 19, American Mathematical Society, 1998. MR 1625845

[16] Fritz John, Partial Differential Equations, 4th ed., Applied Mathematical Sciences, vol. 1, Springer-Verlag, New York, 1982. MR831655 (87g:35002)

[17] J. L. Lions and E. Magénes, Problèmes aux Limites Non Homogènes et Applications, I, Dunod, Paris, 1968. MR0247243

[18] F. Sturm, Modélisation mathématique et numérique d' un problème de propagation en acoustique sous-marine: prise en compte d'un environnement variable tridimensionnel, Thèse de Docteur en Sciences Université de Toulon et du Var, France, 1997.

[19] Fred D. Tappert, The parabolic approximation method, Wave propagation and underwater acoustics (Workshop, Mystic, Conn., 1974), Springer, Berlin, 1977, pp. 224-287. Lecture Notes in Phys., Vol. 70. MR0475274 (57 \#14891)

[20] Vidar Thomée, Galerkin Finite Element Methods for Parabolic Problems, Springer Series in Computational Mathematics, vol. 25, Springer-Verlag, Berlin, 1997. MR 1479170 (98m:65007)

[21] Juan Luis Vázquez and Enzo Vitillaro, Heat equation with dynamical boundary conditions of reactive type, Comm. Partial Differential Equations 33 (2008), no. 4-6, 561-612, DOI 10.1080/03605300801970960. MR2424369 (2009h:35180)

[22] G. E. Zouraris, Analysis of numerical methods for evolution partial differential equations, Ph.D. Thesis, University of Crete, Greece, 1995 (in Greek). 
Department of Mathematics and Applied Mathematics, University of Crete, P.O. Box 2208, GR-710 03 Heraklion, Crete, Greece - and - Institute of Applied and Computational Mathematics, FOrTh, P.O. Box 1527, GR-711 10 Heraklion, Crete, Greece

E-mail address: danton@tem.uoc.gr

Department of Mathematics and Applied Mathematics, University of Crete, P.O. Box 2208, GR-710 03 Heraklion, Crete, Greece - And - Institute of Applied and Computational Mathematics, FOrth, P.O. Box 1527, GR-711 10 Heraklion, Crete, Greece

E-mail address: gkarali@tem.uoc.gr

Department of Mathematics and Applied Mathematics, University of Crete, P.O. Box 2208, GR-710 03 Heraklion, Crete, Greece - and - Institute of Applied and Computational Mathematics, FOrTH, P.O. Box 1527, GR-711 10 Heraklion, Crete, Greece

E-mail address: plex@tem.uoc.gr

Department of Mathematics and Applied Mathematics, University of Crete, P.O. Box 2208, GR-710 03 Heraklion, Crete, Greece - and - Institute of Applied and Computational Mathematics, FORTH, P.O. Box 1527, GR-711 10 Heraklion, Crete, Greece

E-mail address: zouraris@math.uoc.gr 